University of Nebraska - Lincoln

DigitalCommons@University of Nebraska - Lincoln

West Central Research and Extension Center, North Platte

Agricultural Research Division of IANR

2019

\title{
Droplet size and nozzle tip pressure from a pulse-width modulation sprayer
}

Thomas R. Butts

University of Nebraska-Lincoln, tbutts@uaex.edu

Liberty E. Butts

University of Nebraska - Lincoln, libertybutts16@gmail.com

Joe D. Luck

University of Nebraska-Lincoln, jluck2@unl.edu

Bradley K. Fritz

USDA-ARS, brad.fritz@ars.usda.gov

Wesley C. Hoffmann

USDA, Agricultural Research Service, clint.hoffmann@gmail.com

See next page for additional authors

Follow this and additional works at: https://digitalcommons.unl.edu/westcentresext

Part of the Agriculture Commons, Ecology and Evolutionary Biology Commons, and the Plant Sciences Commons

Butts, Thomas R.; Butts, Liberty E.; Luck, Joe D.; Fritz, Bradley K.; Hoffmann, Wesley C.; and Kruger, Greg R., "Droplet size and nozzle tip pressure from a pulse-width modulation sprayer" (2019). West Central Research and Extension Center, North Platte. 114.

https://digitalcommons.unl.edu/westcentresext/114

This Article is brought to you for free and open access by the Agricultural Research Division of IANR at DigitalCommons@University of Nebraska - Lincoln. It has been accepted for inclusion in West Central Research and Extension Center, North Platte by an authorized administrator of DigitalCommons@University of Nebraska Lincoln. 


\section{Authors}

Thomas R. Butts, Liberty E. Butts, Joe D. Luck, Bradley K. Fritz, Wesley C. Hoffmann, and Greg R. Kruger 


\title{
Research Paper
}

\section{Droplet size and nozzle tip pressure from a pulse-width modulation sprayer}

\author{
Thomas R. Butts ${ }^{a, *}$, Liberty E. Butts ${ }^{a}$, Joe D. Luck ${ }^{b}$, Bradley K. Fritz ${ }^{c}$, \\ Wesley C. Hoffmann ${ }^{c}$, Greg R. Kruger ${ }^{a}$ \\ ${ }^{a}$ University of Nebraska-Lincoln, 402 West State Farm Road, North Platte, NE, 69101, USA \\ ${ }^{\mathrm{b}}$ University of Nebraska-Lincoln, P.O. Box 830726, Lincoln, NE, 68583, USA \\ c USDA-ARS Aerial Application Technology Research Unit, 3103 F\&B Road, College Station, TX, 77845, USA
}

\section{A R T I C L E I N F O}

Article history:

Received 23 December 2017

Received in revised form

15 April 2018

Accepted 5 November 2018

Published online 22 November 2018

Keywords:

Duty cycle

non-venturi nozzle

site-specific pest management

solenoid valve

venturi nozzle
Pulse-width modulation (PWM) sprayers can improve application accuracy through flow control, turn compensation, and high-resolution overlap control by pulsing an electronically-actuated solenoid valve which controls the relative proportion of time each solenoid valve is open (duty cycle). The objective of this experiment was to identify the droplet size distribution and nozzle tip pressure when influenced by PWM duty cycle, nozzle technology, and gauge pressure to provide PWM guidelines. The experiment was conducted in a low-speed wind tunnel at the Pesticide Application Technology Laboratory using a SharpShooter ${ }^{\circledR}$ PWM system. In general, for non-venturi nozzles, as duty cycle decreased, droplet size slightly increased between 40 and $100 \%$ duty cycles. Conversely, venturi nozzles did not always follow this trend. The lowest duty cycle evaluated (20\%) negatively impacted droplet size and caused inconsistencies for all nozzle by pressure combinations. The addition of a solenoid valve lowered nozzle tip pressure while gauge pressure remained constant indicating a restriction is present within the solenoid valve. Greater orifice sizes increased the pressure loss observed. Duty cycle minimally impacted nozzle tip pressure trends which were similar to the electrical square wave PWM signals. However, venturi nozzles deviated from this trend, specifically twin-fan, single pre-orifice venturi nozzles. In conclusion, venturi nozzles are not recommended for PWM systems as they may lead to inconsistent applications, specifically in regards to droplet size generation and nozzle tip pressures. Spray pressures of $276 \mathrm{kPa}$ or greater and PWM duty cycles of $40 \%$ or greater are recommended to ensure proper PWM operation.

() 2018 IAgrE. Published by Elsevier Ltd. All rights reserved.

\footnotetext{
* Corresponding author.

E-mail addresses: tbutts@huskers.unl.edu (T.R. Butts), libertybutts16@gmail.com (L.E. Butts), jluck2@unl.edu (J.D. Luck), brad.fritz@ ars.usda.gov (B.K. Fritz), clint.hoffmann@gmail.com (W.C. Hoffmann), gkruger2@unl.edu (G.R. Kruger). 


\section{Nomenclature \\ $a_{0}, \mu \mathrm{m} \quad \mathrm{Y}$-intercept of polynomial regression \\ $a_{n}, \mu \mathrm{m} \quad$ Constant coefficients of polynomial regression

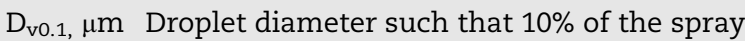 volume was contained in droplets < stated diameter \\ $\mathrm{D}_{\mathrm{v} 0.5}, \mu \mathrm{m}$ Droplet diameter such that $50 \%$ of the spray volume was contained in droplets < stated diameter \\ $D_{\mathrm{v} 0.9}, \mu \mathrm{m}$ Droplet diameter such that $90 \%$ of the spray volume was contained in droplets < stated diameter \\ driftable fines, \% Spray volume with droplets $\leq 150 \mu \mathrm{m}$ duty cycle, \% Relative proportion of time solenoid valve is open \\ $\mathrm{x}, \% \quad$ Duty cycle \\ AMS Ammonium sulphate \\ ANOVA Analysis of variance \\ LSD Least significant difference \\ PWM Pulse-width modulation \\ $r^{2} \quad$ Coefficient of determination}

the importance of droplet size on drift mitigation (Bueno, da Cunha, \& de Santana, 2017; Hewitt, 1997; Johnson, Roeth, Martin, \& Klein, 2006) and herbicide efficacy (Etheridge, Womac, \& Mueller, 1999; Knoche, 1994; Meyer, Norsworthy, Kruger, \& Barber, 2016). Furthermore, homogenisation of the droplet sizes represented within a spray pattern coupled with reduced droplet velocities could result in greater droplet adhesion to leaf surfaces and increase biological efficacy, while limiting drift potential (De Cock, Massinon, Salah, \& Lebeau, 2017).

Pulse-width modulation (PWM) sprayers allow for several factors, including application pressure and spray droplet size, to be standardized across a range of sprayer speeds while variably controlling flow to increase application precision. Flow is controlled by pulsing an electronically-actuated solenoid valve placed directly upstream of the nozzle (Giles \& Comino, 1989). The flow is changed by controlling the relative proportion of time each solenoid valve is open (duty cycle). This system allows real-time flow rate changes to be made without manipulating application pressure as in other variable rate spray application systems (Anglund \& Ayers, 2003). Additionally, PWM solenoid valves buffer some negative impacts, such as spray boom velocity variation during turning movements and flow on/off latency of automatic boom shutoffs, observed with other rate controller systems (Luck, Sharda, Pitla, Fulton, \& Shearer, 2011; Sharda, Fulton, McDonald, \& Brodbeck, 2011; Sharda, Luck, Fulton, McDonald, \& Shearer, 2013). Application pressure based variable rate flow control devices have been shown to have slow response time and affect nozzle performance, specifically droplet size (Giles \& Comino, 1989). Previous PWM research illustrated little to no effect from duty cycle on spray droplet size (Giles \& Comino, 1990; Giles, Henderson, \& Funk, 1996); however, only non-venturi nozzles and nozzles lacking a preorifice were evaluated.

PWM sprayers provide the possibility for more precise applications through automatic boom and individual nozzle shut off controls (Luck, Pitla, et al., 2010; Luck, Zandonadi, Luck, \& Shearer, 2010) and minimizing changes in droplet trajectory and velocity (Butts, Hoffmann, Luck, \& Kruger, 2017; Giles, 2001; Giles \& Ben-Salem, 1992). Furthermore, pulsing dual nozzle configurations increased coverage of Palmer amaranth (Amaranthus palmeri S. Wats.) while simultaneously minimizing the drift potential of small droplets (Womac et al., 2017; Womac, Melnichenko, Steckel, Montgomery, \& Hayes, 2016). One drawback to PWM application systems has been the inability to create wide ranges of droplet distributions because venturi nozzles are not recommended (Capstan Ag Systems Inc., 2013). However, previous research demonstrated there are commercially available, non-venturi nozzles that can produce the range of droplet size distributions needed to reduce drift potential (Butts, Geyer, \& Kruger, 2015).

Current nozzle technologies and application parameters must be evaluated on PWM sprayers to determine best use practices for the equipment. The objective of this experiment was to identify the droplet size distribution and pressure at the nozzle tip as influenced by PWM duty cycle, current nozzle technology (venturi versus non-venturi), and gauge application pressure, and provide guidelines for optimal PWM use. 


\section{Materials and methods}

\subsection{Experimental design}

Research was conducted in the spring and summer of 2016 to evaluate the effect of nozzle type, PWM duty cycle, and gauge application pressure on droplet size distribution and nozzle tip pressure. The experiment was conducted using the lowspeed wind tunnel at the Pesticide Application Technology Laboratory located at the West Central Research and Extension Center in North Platte, NE. Creech et al. (2015) and Henry, Kruger, Fritz, Hoffmann, and Bagley (2014) provide further details regarding the low-speed wind tunnel framework and operation. The wind tunnel was equipped with a SharpShooter ${ }^{\circledR}$ PWM system (Capstan Ag Systems, Inc., Topeka, KS) to select the specific duty cycle for each treatment.

The experiment was a $12 \times 6 \times 3 \times 2$ factorial cumulating in a total of 432 treatments, and each treatment was replicated three times (three separate nozzle traverses across the laser). The treatments consisted of 12 nozzle types, 6 PWM duty cycles, 3 gauge application pressures (pressure before the solenoid valve), and 2 spray solutions (Table 1). Droplet size and nozzle tip pressure of water were also measured for the 12 nozzle types at the 3 gauge application pressures in a standard nozzle body configuration (no solenoid valve). Glyphosate (Roundup PowerMAX®, Monsanto Co., St. Louis, MO 63167) plus ammonium sulphate (AMS) solution was applied at $0.87 \mathrm{~kg}$ ae ha ${ }^{-1}$ and $1.91 \mathrm{~kg} \mathrm{ha}^{-1}$, respectively, in a carrier volume of $94 \mathrm{~L} \mathrm{ha}^{-1}$ to assess whether an active ingredient within the spray solution would affect droplet size and nozzle tip pressure trends when pulsed compared to water alone. Reference nozzles were used to determine spray classifications (ASABE, 2009) and allow for comparisons between testing laboratories (Fritz et al., 2014). Air temperature, solution temperature, and relative humidity were also recorded during the time periods the experiment was conducted.

\subsection{Droplet size distribution collection}

The droplet size distribution for each treatment was measured using a Sympatec HELOS-VARIO/KR laser diffraction system with the R7 lens (Sympatec Inc., Clausthal, Germany). The laser was linked with WINDOX 5.7.0.0 software (Sympatec Inc.) operated on a computer adjacent to the laser. The R7 lens measures droplets in a dynamic size range from 18 to $3500 \mu \mathrm{m}$. The laser consists of two main components, an emitter housing containing the optical box and the source of the laser, and a receiver housing containing the lens and detector element (Fig. 1). The two laser housings are separated $(1.2 \mathrm{~m})$ on each side of the wind tunnel and mounted on an aluminum optical bench rail that was connected underneath the wind tunnel to maintain proper laser alignment. The laser was beamed through two $10-\mathrm{cm}$ holes bored into the Plexiglass wind tunnel side wall. The spray plume was oriented perpendicular to the laser and traversed at $0.2 \mathrm{~m} \mathrm{~s}^{-1}$ using a mechanical linear actuator. The distance from the nozzle tip to the laser was $30 \mathrm{~cm}$. The wind tunnel generated a $24 \mathrm{~km} \mathrm{~h}^{-1}$ airspeed in which measurements were recorded (Fritz, Hoffmann, Bagley, et al., 2014). The laser diffraction system provided multiple categories to compare the spray droplet distributions of each treatment. The treatments in this study were compared using the $D_{\mathrm{v} 0.1}, D_{\mathrm{v} 0.5}$, and $D_{\mathrm{v} 0.9}$ parameters which represent the droplet diameters such that 10,50 , and $90 \%$ of the spray volume was contained in droplets of smaller diameter, respectively. Furthermore, the percent of spray volume with droplets $\leq 150 \mu \mathrm{m}$ [referred to as driftable fines throughout (Hewitt, 1997)] were recorded for each treatment.

Table 1 - Nozzles (12), pulse-width modulation duty cycles (7), gauge application pressures (3), and spray solutions (2) evaluated in a factorial arrangement of treatments in this research.

\begin{tabular}{|c|c|c|c|c|c|}
\hline \multicolumn{3}{|c|}{ Broadcast nozzles } & \multirow[b]{2}{*}{ Duty cycle \% } & \multirow[b]{2}{*}{ Gauge pressure $\mathrm{kPa}$} & \multirow[b]{2}{*}{ Spray solution } \\
\hline Abbreviation & Name & Design & & & \\
\hline AITTJ-6011004 & Air Induction Turbo TwinJet & Venturi & Standard ${ }^{\mathrm{e}}$ & 207 & Water alone \\
\hline $\mathrm{AM}_{11002^{\mathrm{b}}}$ & Airmix & Venturi & 100 & 276 & $\begin{array}{c}\text { Glyphosate plus ammonium } \\
\text { sulphate (AMS) }\end{array}$ \\
\hline AM11004 ${ }^{b}$ & Airmix & Venturi & 80 & 414 & \\
\hline AMDF11004 & Airmix DualFan & Venturi & 60 & & \\
\hline AMDF11008 & Airmix DualFan & Venturi & 50 & & \\
\hline GAT11004 & GuardianAIR Twin & Venturi & 40 & & \\
\hline TTI11004 & Turbo Teejet Induction & Venturi & 20 & & \\
\hline DR11004 & Combo-Jet Drift Control & Non-Venturi & & & \\
\hline ER11004 & Combo-Jet Extended Range & Non-Venturi & & & \\
\hline MR11004 & Combo-Jet Mid Range & Non-Venturi & & & \\
\hline SR11004 & Combo-Jet Small Reduction & Non-Venturi & & & \\
\hline UR11004 & Combo-Jet Ultra Drift Control & Non-Venturi & & & \\
\hline \multicolumn{6}{|c|}{$\begin{array}{l}\text { a TeeJet Technologies, Spraying Systems Co., Glendale Heights, IL, USA. } \\
\text { b Greenleaf Technologies, Covington, LA, USA. } \\
\text { c Pentair Hypro SHURflo plc., Minneapolis, MN, USA. } \\
\text { d Wilger Industries Ltd., Lexington, TN, USA. } \\
\text { e Standard duty cycle indicates no solenoid valve is equipped. } \\
\text { f Glyphosate (Roundup PowerMAX®, Monsanto Co., St. Louis, MO, USA) plus ammonium sulphate (AMS) solution was applied at } 0.87 \mathrm{~kg}^{-1} \text { ae ha }{ }^{-1} \\
\text { and } 1.91 \mathrm{~kg} \mathrm{ha}^{-1} \text {, respectively, in a carrier volume of } 941 \mathrm{ha}^{-1} \text {. }\end{array}$} \\
\hline
\end{tabular}




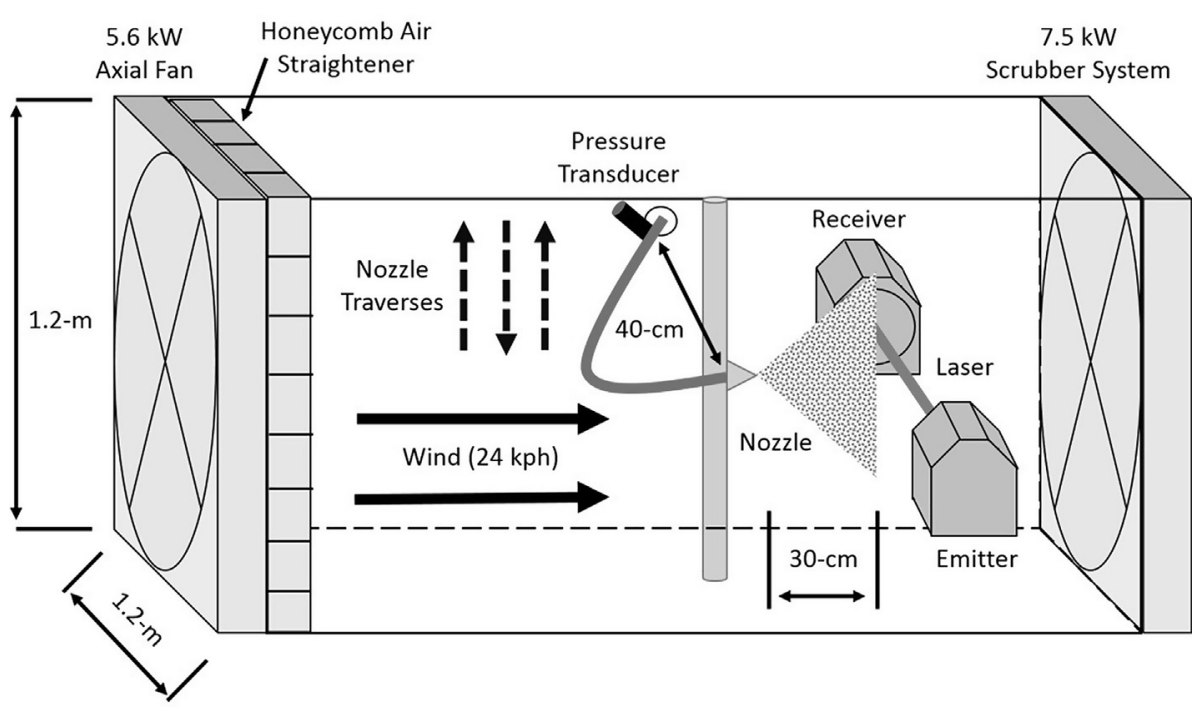

Fig. 1 - Illustration of the low-speed wind tunnel and laser diffraction system used for droplet spectrum analysis at the University of Nebraska-Lincoln Pesticide Application Technology Laboratory located in North Platte, NE.

\subsection{Nozzle tip pressure determination}

The gauge application pressures of 207,276 , and $414 \mathrm{kPa}$ were verified by a PX309, 5 V, 0-689 kPa range pressure transducer (Omega Engineering, Inc., Stamford, CT) located $40 \mathrm{~cm}$ upstream from the solenoid valve and connected to a display monitor. The nozzle tip pressure was measured using a similar pressure transducer installed inline between the PWM solenoid valve and nozzle (Fig. 2). The nozzle tip pressure transducer was powered by an $80 \mathrm{~W}$ switching mode DC power supply (Extech Instruments, Nashua, $\mathrm{NH}$ ) which was set to output $10 \mathrm{~V}$. These specific pressure transducers have a silicon sensor protected by a fluid filled stainless steel diaphragm that converts pressure to an analogue electrical signal. The analogue electrical signals were sampled at a $100 \mathrm{~Hz}$ rate for $5 \mathrm{~s}$ using an Arduino Mega 2560 board (opensource prototyping platform, Arduino.cc). The Arduino board converted the analogue signals to digital and sent them to a serial monitor on a connected computer where the signals were transformed to pressure measurements $(\mathrm{kPa})$.

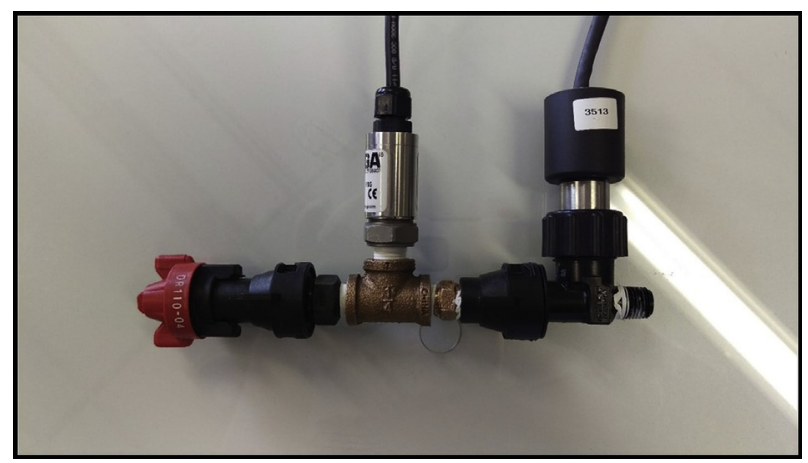

Fig. 2 - Nozzle body and pressure transducer assembly used to measure nozzle tip pressures after the pulse-width modulation solenoid valve. Another pressure transducer was connected inline $40-\mathrm{cm}$ upstream from this assembly to provide gauge application pressure.

\subsection{Statistical analyses}

Regression analysis was conducted on $D_{\mathrm{v} 0.5}$ values to allow for droplet size predictions as impacted by duty cycle within nozzle type and gauge application pressure and evaluate the variability across nozzle types when pulsed. Seventy different linear, nonlinear, and polynomial models were evaluated to determine best fit using CurveExpert Professional ${ }^{\odot}$ (v. 2.6.5, Hyams Development). Droplet size parameters, driftable fines, and average nozzle tip pressure data were subjected to analysis of variance (ANOVA) using a mixed effect model in SAS (SAS v9.4, SAS Institute Inc., Cary, NC, USA). Nozzle type, PWM duty cycle, gauge application pressure, and spray solution were treated as fixed effects. Means were separated using Fisher's protected LSD test with the Tukey adjustment to correct for multiplicity. A gamma distribution was used for analysis of droplet size parameters and nozzle tip pressures as data were bound between zero and positive infinity, and a beta distribution was used for analysis of driftable fines as data were bound between zero and one (Stroup, 2013). Backtransformed data are presented for clarity.

\section{Results and discussion}

The environmental conditions within the Pesticide Application Technology Laboratory were maintained to be relatively constant. The average air temperature and relative humidity throughout the duration of this study was $25 \mathrm{C}$ and $47 \%$, respectively. The average solution temperature across treatments was $21 \mathrm{C}$. Previous literature suggested less than $5 \mathrm{C}$ difference between air and solution temperatures to minimize variance in droplet size measurements (Hoffmann, Fritz, \& Martin, 2011; Miller \& Tuck, 2005).

The $D_{\mathrm{v} 0.5}$ regression over duty cycle analysis revealed that a polynomial regression model (Equation (1)) was among the top fitting models across pressures and nozzles; therefore it was fit to all data. The degree of polynomial (first through 

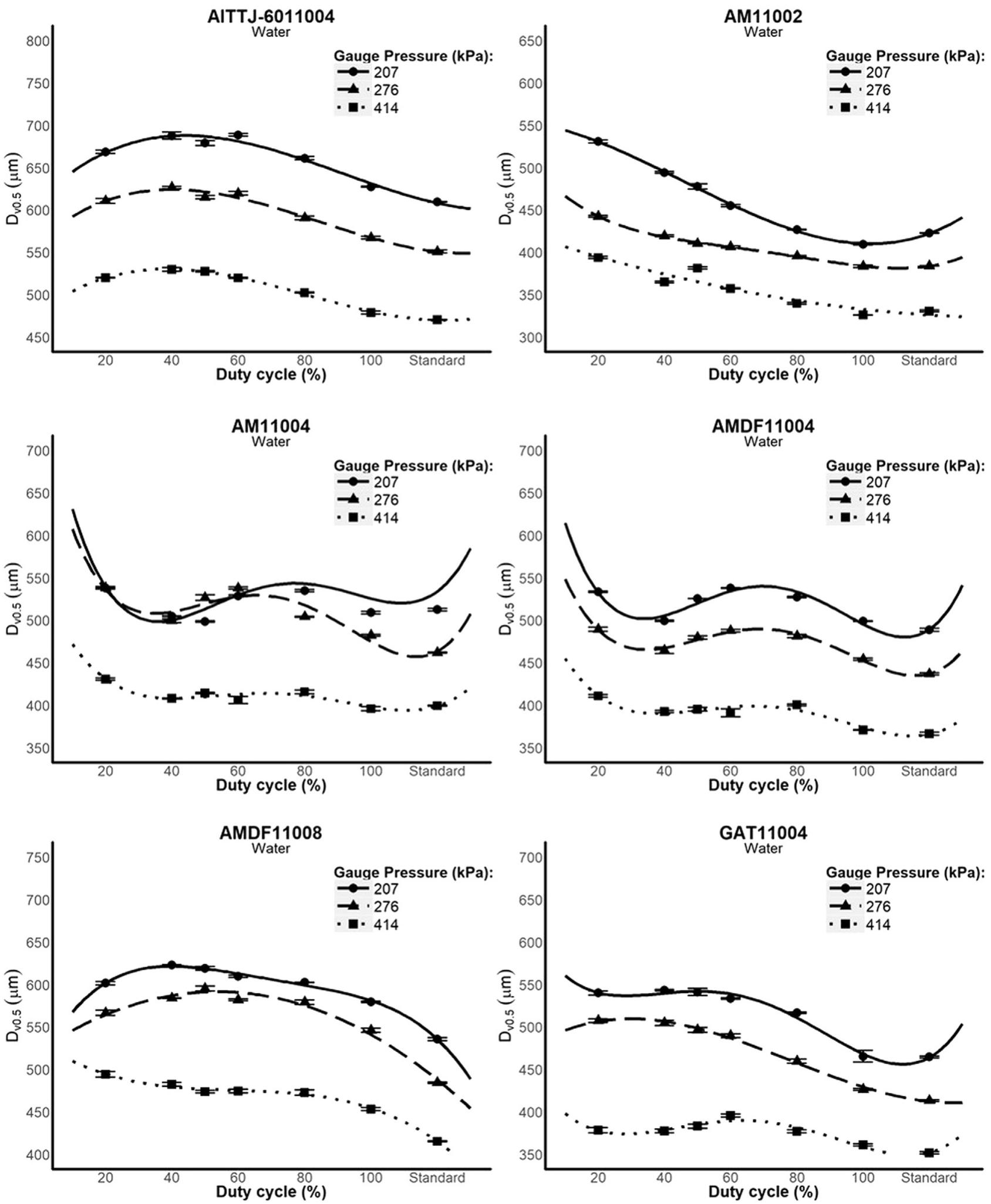

Fig. 3 - Polynomial regressions of droplet size data $\left(D_{\mathrm{v} 0.5}\right)$ of water as influenced by duty cycle for the AITTJ-6011004 (top left), AM11002 (top right), AM11004 (middle left), AMDF11004 (middle right), AMDF11008 (bottom left), and GAT11004 (bottom right) nozzles. 

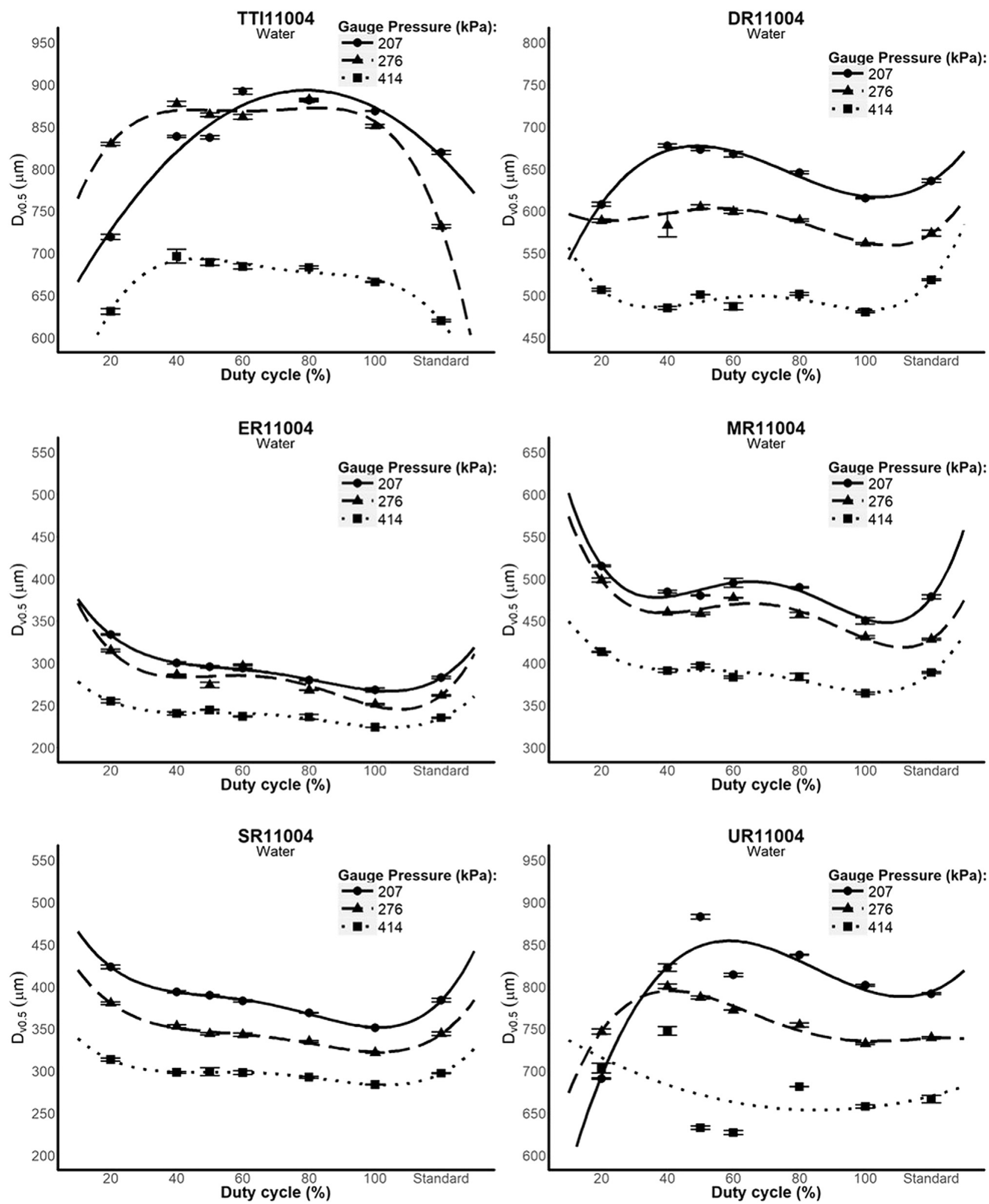

Fig. 4 - Polynomial regressions of droplet size data $\left(D_{\mathrm{vo.5}}\right)$ of water as influenced by duty cycle for the TTI11004 (top left), DR11004 (top right), ER11004 (middle left), MR11004 (middle right), SR11004 (bottom left), and UR11004 (bottom right) nozzles. 
fourth degrees) for each treatment was selected based on both the AICC and an F-test at $\alpha=0.01$.

$D_{v 0.5}=a_{n} x^{n}+a_{n-1} x^{n-1}+\ldots+a_{2} x^{2}+a_{1} x+a_{0}$

Where $D_{v 0.5}$ is droplet diameter such that $50 \%$ of the spray volume was contained in droplets of diameter less than the stated diameter, $a_{0}$ is y-intercept, $a_{n}$ is constant coefficients, and $x$ is duty cycle.

Across all response variables, ANOVA resulted in a nozzle*duty cycle*gauge application pressure*solution interaction $(\mathrm{P}<0.0001)$. Therefore, comparisons were reduced to strictly observe the effect of PWM duty cycle on droplet size $\left(D_{\mathrm{v} 0.1}\right.$, $D_{\mathrm{v} 0.5}, D_{\mathrm{v} 0.9}$, and driftable fines) within a nozzle, gauge application pressure, and solution. Moreover, for nozzle tip pressure measurements, comparisons were reduced to specifically observe the effect of nozzle type within a solution, gauge application pressure, and PWM duty cycle. Relative trends across analyses were similar for the water and glyphosate plus AMS solutions (data not shown); therefore, the water solution is strictly discussed within this manuscript.

\subsection{Droplet size}

\subsubsection{Venturi nozzles}

Polynomial regressions established for venturi nozzles (AITTJ6011004, AM11002, AM11004, AMDF11004, AMDF11008,

Table 2 - Polynomial regression parameters $\left(a_{0}, a_{1}, a_{2}, a_{3}, a_{4}\right)$ and coefficient of determination $\left(r^{2}\right)$ for droplet size $\left(D_{\mathrm{v} 0.5}\right)$ regressed over duty cycle of water for each nozzle*pressure combination.

\begin{tabular}{|c|c|c|c|c|c|c|c|}
\hline \multirow[t]{2}{*}{ Nozzle } & $\begin{array}{c}\text { Gauge } \\
\text { pressure }\end{array}$ & $a_{0}$ & $a_{1}$ & $a_{2}$ & $a_{3}$ & $a_{4}$ & \multirow{2}{*}{$\begin{array}{c}\begin{array}{c}\text { Coefficient of } \\
\text { determination }\end{array} \\
r^{2}\end{array}$} \\
\hline & $\mathrm{kPa}$ & \multicolumn{5}{|c|}{$-\mu \mathrm{m}-$} & \\
\hline $\begin{array}{l}\text { AITTJ- } \\
\quad 6011004^{\mathrm{a}}\end{array}$ & 207 & 612.06 & 3.85 & -0.06 & 2.11 E -04 & - & 0.96 \\
\hline AM11002 & 207 & 552.89 & -0.63 & -0.03 & 2.07 E -04 & - & 0.99 \\
\hline AM11004 & 207 & 803.43 & -22.31 & 0.56 & -5.47 E -03 & 1.85 E -05 & 0.86 \\
\hline AMDF11004 & 207 & 777.85 & -21.41 & 0.57 & $-5.81 \mathrm{E}-03$ & 2.02 E -05 & 0.94 \\
\hline AMDF11008 & 207 & 506.68 & 7.70 & -0.18 & $1.70 \mathrm{E}-03$ & 6.06 E -06 & 0.99 \\
\hline GAT11004 & 207 & 608.88 & -6.74 & 0.22 & $-2.66 \mathrm{E}-03$ & 1.05 E -05 & 0.97 \\
\hline TTI11004 & 207 & 595.66 & 7.51 & -0.05 & - & - & 0.94 \\
\hline DR11004 & 207 & 446.70 & 11.17 & -0.17 & 7.33 E -04 & - & 0.97 \\
\hline ER11004 & 207 & 448.53 & -9.13 & 0.21 & $-2.11 \mathrm{E}-03$ & 7.65 E -06 & 0.98 \\
\hline MR11004 & 207 & 767.14 & -21.60 & 0.56 & $-5.87 \mathrm{E}-03$ & $2.11 \mathrm{E}-05$ & 0.91 \\
\hline SR11004 & 207 & 540.98 & -9.65 & 0.24 & -2.57 E -03 & 9.84 E -06 & 0.99 \\
\hline UR11004 ${ }^{c}$ & 207 & 422.63 & 17.85 & -0.23 & 9.12 E -04 & - & 0.86 \\
\hline $\begin{array}{l}\text { AITTJ- } \\
\quad 6011004^{\mathrm{a}}\end{array}$ & 276 & 563.95 & 3.35 & -0.05 & $2.18 \mathrm{E}-04$ & - & 0.97 \\
\hline $\mathrm{AM}_{11002^{\mathrm{b}}}$ & 276 & 503.11 & -4.46 & 0.09 & $-8.49 \mathrm{E}-04$ & 3.00 E -06 & 0.99 \\
\hline AM11004 & 276 & 747.43 & -18.38 & 0.49 & $-5.06 \mathrm{E}-03$ & 1.77 E -05 & 0.89 \\
\hline AMDF11004 & 276 & 665.57 & -15.31 & 0.40 & -4.07 E -03 & $1.39 \mathrm{E}-05$ & 0.96 \\
\hline AMDF11008 & 276 & 522.78 & 2.55 & -0.02 & - & - & 0.97 \\
\hline GAT11004 ${ }^{\mathrm{d}}$ & 276 & 476.70 & 2.41 & -0.05 & $2.13 \mathrm{E}-04$ & - & 0.99 \\
\hline TTI11004 & 276 & 642.98 & 15.78 & -0.40 & 4.37 E -03 & -1.74 E -05 & 0.98 \\
\hline DR11004 & 276 & 624.47 & -4.10 & 0.15 & -1.96 E -03 & 7.96 E -06 & 0.94 \\
\hline ER11004 & 276 & 475.18 & -13.43 & 0.34 & $-3.50 \mathrm{E}-03$ & $1.26 \mathrm{E}-05$ & 0.89 \\
\hline MR11004 & 276 & 715.79 & -18.31 & 0.46 & $-4.65 \mathrm{E}-03$ & 1.62 E -05 & 0.96 \\
\hline SR11004 & 276 & 487.27 & -8.52 & 0.20 & $-2.03 \mathrm{E}-03$ & 7.46 E -06 & 0.97 \\
\hline UR11004 ${ }^{c}$ & 276 & 550.55 & 15.30 & -0.32 & $2.60 \mathrm{E}-03$ & -7.28 E -06 & 0.96 \\
\hline $\begin{array}{l}\text { AITTJ- } \\
6011004^{a}\end{array}$ & 414 & 479.36 & 2.94 & -0.05 & 2.07 E -04 & - & 0.99 \\
\hline AM11002 & 414 & 419.10 & -1.30 & 0.04 & - & - & 0.89 \\
\hline AM11004 & 414 & 546.59 & -9.56 & 0.23 & $-2.29 \mathrm{E}-03$ & 7.86 E -06 & 0.82 \\
\hline AMDF11004 & 414 & 536.24 & -10.51 & 0.26 & $-2.64 \mathrm{E}-03$ & 9.00 E -06 & 0.89 \\
\hline AMDF11008 ${ }^{b}$ & 414 & 532.04 & -2.62 & 0.04 & $-2.36 \mathrm{E}-04$ & - & 0.98 \\
\hline GAT11004 ${ }^{\mathrm{d}}$ & 414 & 445.16 & -6.41 & 0.19 & -2.16 E -03 & 7.89 E -06 & 0.90 \\
\hline TTI11004 & 414 & 401.07 & 18.21 & -0.40 & 3.79 E -03 & $-1.30 \mathrm{E}-05$ & 0.95 \\
\hline DR11004 & 414 & 654.99 & -12.86 & 0.34 & $-3.60 \mathrm{E}-03$ & 1.32 E -05 & 0.74 \\
\hline ER11004c & 414 & 321.09 & -5.54 & 0.14 & -1.48 E -03 & $5.45 \mathrm{E}-06$ & 0.89 \\
\hline MR11004 & 414 & 516.55 & -8.66 & 0.22 & $-2.36 \mathrm{E}-03$ & 8.83 E -06 & 0.89 \\
\hline SR11004 & 414 & 385.76 & -6.06 & 0.15 & $-1.61 \mathrm{E}-03$ & $5.93 \mathrm{E}-06$ & 0.88 \\
\hline UR11004 ${ }^{c}$ & 414 & 759.89 & -2.48 & 0.01 & - & - & 0.25 \\
\hline $\begin{array}{l}\text { a } \text { TeeJet Tech } \\
\text { b Greenleaf T } \\
\text { c Wilger Indu } \\
\text { d Pentair Hyp }\end{array}$ & $\begin{array}{l}\text { nologies, S } \\
\text { echnologie } \\
\text { stries Ltd., } \\
\text { ro SHURflo }\end{array}$ & $\begin{array}{l}\text { ig Syste } \\
\text { ington, } \\
\text { gton, Tl } \\
\text { Minnea }\end{array}$ & $\begin{array}{l}\text { lendale } \\
\text { USA. }\end{array}$ & L, USA. & & & \\
\hline
\end{tabular}


GAT11004, and TTI11004) to predict the effect of duty cycle on the $D_{\mathrm{v} 0.5}$ for each gauge pressure are presented in Figs. 3 and 4 . The $20 \%$ duty cycle caused severe deviations from observed droplet size trends across other duty cycle treatments (Figs. 3 and 4) resulting in curved tails to the fit models. This duty cycle was determined as the cause of the required polynomial regression as opposed to linear models previously used in PWM droplet size research (Giles \& Comino, 1990). It is highly recommended that applicators operate a PWM sprayer at $40 \%$ duty cycles or greater. The resulting model parameters and coefficient of determination $\left(r^{2}\right)$ values are presented in Table 2. Generally, as duty cycle decreased, the droplet size increased across venturi nozzles within each gauge pressure. On average, as duty cycle decreased from 100 to $40 \%$, models predicted an increase in droplet size of $0.90,0.64$, and $0.48 \mu \mathrm{m}$ for every $1 \%$ duty cycle decrease for the 207, 276, and $414 \mathrm{kPa}$ gauge pressures, respectively, across venturi nozzles. Although the $r^{2}$ values tended to decrease as gauge pressure increased, these results indicate increasing the operating pressure on PWM sprayers can buffer the effect of pulsing on droplet size.

The droplet size distributions and driftable fines of venturi nozzles as affected by pulsing are presented in Table 3 through 6. Across duty cycles, the droplet size distributions from venturi nozzles followed the pattern (from smallest to greatest): AM11002 < GAT11004 < AMDF11004 < AM1 $1004<$ AMDF11008 < AITTJ-6011004 < TTI11004 (Tables 3-5).

Table 3 - Droplet size data such that $10 \%$ of the spray volume is contained in droplets of lesser diameter $\left(D_{\mathrm{v0.1}}\right)$ for water impacted by duty cycle for nozzle and pressure combinations.

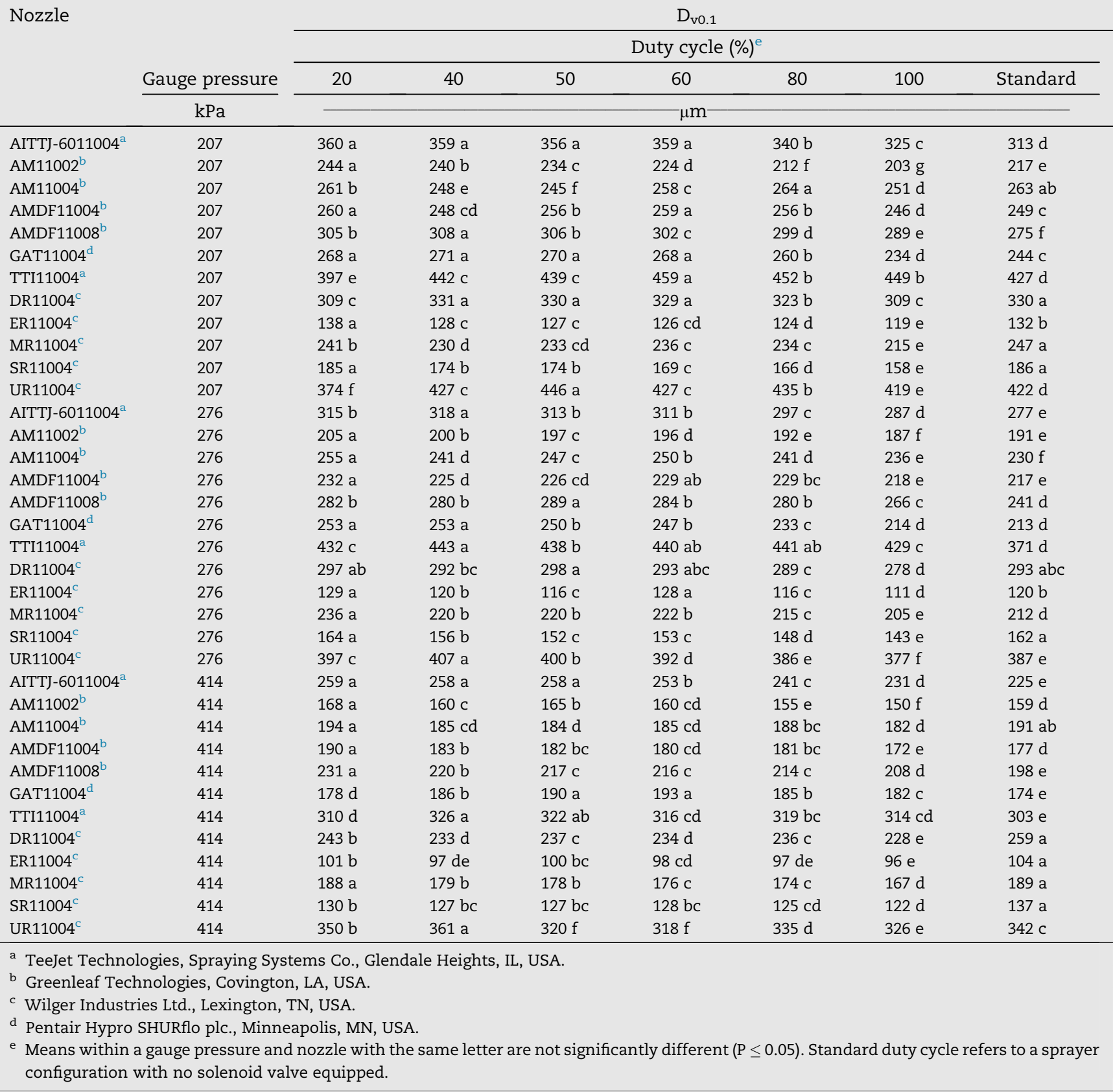


Table 4 - Droplet size data such that $\mathbf{5 0 \%}$ of the spray volume is contained in droplets of lesser diameter $\left(D_{\mathrm{v} 0.5}\right)$ for water impacted by duty cycle for nozzle and pressure combinations.

\begin{tabular}{|c|c|c|c|c|c|c|c|c|}
\hline \multirow[t]{4}{*}{ Nozzle } & \multirow[b]{3}{*}{ Gauge pressure } & \multicolumn{7}{|c|}{$\mathrm{D}_{\mathrm{v} 0.5}$} \\
\hline & & \multicolumn{7}{|c|}{ Duty cycle $(\%)^{\mathrm{e}}$} \\
\hline & & 20 & 40 & 50 & 60 & 80 & 100 & Standard \\
\hline & $\mathrm{kPa}$ & \multicolumn{7}{|c|}{$-\mu \mathrm{m}-$} \\
\hline AITTJ-6011004 & 207 & $669 c$ & $688 \mathrm{a}$ & $679 \mathrm{~b}$ & 689 a & $661 \mathrm{~d}$ & $627 \mathrm{e}$ & $609 \mathrm{f}$ \\
\hline AM11002 ${ }^{b}$ & 207 & $531 \mathrm{a}$ & $494 \mathrm{~b}$ & $478 \mathrm{c}$ & $455 d$ & $427 \mathrm{e}$ & $409 \mathrm{~g}$ & $423 \mathrm{f}$ \\
\hline AM11004 & 207 & $538 \mathrm{a}$ & $505 \mathrm{f}$ & $498 \mathrm{~g}$ & $529 c$ & $535 \mathrm{~b}$ & 509 e & $512 \mathrm{~d}$ \\
\hline AMDF11004 ${ }^{b}$ & 207 & $533 \mathrm{~b}$ & $499 \mathrm{~d}$ & $525 c$ & 538 a & $527 c$ & $499 \mathrm{~d}$ & 489 e \\
\hline AMDF11008 & 207 & $601 \mathrm{~d}$ & $623 \mathrm{a}$ & $619 b$ & $610 c$ & $602 \mathrm{~d}$ & 579 e & $536 \mathrm{f}$ \\
\hline GAT11004 & 207 & $540 \mathrm{ab}$ & $543 \mathrm{a}$ & $541 \mathrm{ab}$ & $534 b$ & $517 \mathrm{c}$ & $465 \mathrm{~d}$ & $465 d$ \\
\hline TTI11004 & 207 & $719 \mathrm{f}$ & $838 d$ & 837 d & $892 \mathrm{a}$ & $882 \mathrm{~b}$ & $868 c$ & 819 e \\
\hline DR11004 & 207 & $608 \mathrm{f}$ & $677 \mathrm{a}$ & $673 \mathrm{a}$ & $667 \mathrm{~b}$ & $646 c$ & $615 \mathrm{e}$ & $636 \mathrm{~d}$ \\
\hline ER11004 & 207 & $334 \mathrm{a}$ & $300 \mathrm{~b}$ & 296 bc & $294 \mathrm{C}$ & $280 \mathrm{~d}$ & $268 \mathrm{e}$ & $283 d$ \\
\hline MR11004 & 207 & $515 \mathrm{a}$ & $484 \mathrm{~cd}$ & $480 \mathrm{~d}$ & $495 \mathrm{~b}$ & $490 \mathrm{bc}$ & $450 \mathrm{e}$ & $478 d$ \\
\hline SR11004 & 207 & $423 \mathrm{a}$ & $394 b$ & $390 \mathrm{c}$ & $383 d$ & 369 e & $351 \mathrm{f}$ & $384 \mathrm{~d}$ \\
\hline UR11004 & 207 & $691 \mathrm{~g}$ & $822 \mathrm{c}$ & 883 a & $814 d$ & $838 \mathrm{~b}$ & 801 e & $792 \mathrm{f}$ \\
\hline AITTJ-6011004 & 276 & $611 \mathrm{c}$ & $626 \mathrm{a}$ & $615 \mathrm{bc}$ & $620 \mathrm{~b}$ & $591 \mathrm{~d}$ & 567 e & $551 \mathrm{f}$ \\
\hline AM11002 ${ }^{b}$ & 276 & $442 \mathrm{a}$ & $419 b$ & $410 \mathrm{c}$ & $406 d$ & $396 \mathrm{e}$ & $383 \mathrm{f}$ & $384 \mathrm{f}$ \\
\hline AM11004 & 276 & 538 a & $499 \mathrm{~d}$ & $526 \mathrm{~b}$ & 538 a & $504 \mathrm{c}$ & $482 \mathrm{e}$ & $462 \mathrm{f}$ \\
\hline AMDF11004 & 276 & $489 \mathrm{a}$ & $464 \mathrm{c}$ & $480 \mathrm{~b}$ & $488 \mathrm{a}$ & $481 \mathrm{~b}$ & $454 \mathrm{~d}$ & $437 \mathrm{e}$ \\
\hline AMDF11008 & 276 & $567 \mathrm{c}$ & $584 \mathrm{~b}$ & 595 a & $582 \mathrm{~b}$ & $579 \mathrm{~b}$ & $546 \mathrm{~d}$ & $484 \mathrm{e}$ \\
\hline GAT11004 & 276 & 507 a & 505 a & $496 \mathrm{~b}$ & $490 \mathrm{c}$ & $460 \mathrm{~d}$ & $426 \mathrm{e}$ & $413 \mathrm{f}$ \\
\hline TTI11004 & 276 & $829 d$ & $877 \mathrm{a}$ & $864 \mathrm{~b}$ & 862 b & $882 \mathrm{a}$ & $851 \mathrm{c}$ & $732 \mathrm{e}$ \\
\hline DR11004 & 276 & $588 \mathrm{bc}$ & $583 \mathrm{~cd}$ & $605 \mathrm{a}$ & $599 \mathrm{ab}$ & 589 bc & $561 \mathrm{e}$ & $574 \mathrm{de}$ \\
\hline ER11004 & 276 & 315 a & $286 \mathrm{c}$ & $274 d$ & $296 \mathrm{~b}$ & 268 e & $251 \mathrm{~g}$ & $262 \mathrm{f}$ \\
\hline MR11004 & 276 & $498 \mathrm{a}$ & $460 \mathrm{c}$ & $458 \mathrm{c}$ & $477 \mathrm{~b}$ & $457 c$ & $431 \mathrm{~d}$ & $428 d$ \\
\hline SR11004 & 276 & $380 \mathrm{a}$ & $353 b$ & $344 \mathrm{c}$ & $343 c$ & $335 \mathrm{~d}$ & $321 \mathrm{e}$ & $344 \mathrm{c}$ \\
\hline UR11004 ${ }^{c}$ & 276 & $746 \mathrm{e}$ & $800 \mathrm{a}$ & $787 \mathrm{~b}$ & $772 \mathrm{c}$ & $755 \mathrm{~d}$ & $732 \mathrm{~g}$ & $739 \mathrm{f}$ \\
\hline AITTJ-6011004 & 414 & $520 \mathrm{~b}$ & $530 \mathrm{a}$ & $527 \mathrm{a}$ & $520 \mathrm{~b}$ & $502 \mathrm{c}$ & $479 \mathrm{~d}$ & $470 \mathrm{e}$ \\
\hline AM11002 $2^{b}$ & 414 & $394 \mathrm{a}$ & $365 c$ & $381 \mathrm{~b}$ & $357 d$ & 340 e & $326 \mathrm{~g}$ & $331 \mathrm{f}$ \\
\hline AM11004 & 414 & $431 \mathrm{a}$ & $408 c$ & $414 \mathrm{~b}$ & $406 \mathrm{c}$ & $416 \mathrm{~b}$ & $396 \mathrm{~d}$ & $399 \mathrm{~d}$ \\
\hline AMDF11004 ${ }^{b}$ & 414 & $411 \mathrm{a}$ & $393 c$ & 395 bc & $391 \mathrm{c}$ & $400 \mathrm{~b}$ & $371 \mathrm{~d}$ & $366 \mathrm{~d}$ \\
\hline AMDF11008 ${ }^{b}$ & 414 & $494 \mathrm{a}$ & $482 \mathrm{~b}$ & $474 \mathrm{c}$ & $474 \mathrm{c}$ & $473 c$ & $453 d$ & $415 \mathrm{e}$ \\
\hline GAT11004 & 414 & $378 \mathrm{bc}$ & $377 c$ & $383 \mathrm{~b}$ & 396 a & $377 c$ & $361 \mathrm{~d}$ & $352 \mathrm{e}$ \\
\hline TTI11004 & 414 & $631 \mathrm{~d}$ & $696 \mathrm{a}$ & $689 \mathrm{ab}$ & $684 \mathrm{~b}$ & $683 b$ & $666 c$ & $620 \mathrm{e}$ \\
\hline DR11004 & 414 & $506 \mathrm{~b}$ & $485 d$ & $501 c$ & $487 d$ & $501 \mathrm{c}$ & $480 \mathrm{e}$ & 518 a \\
\hline ER11004 & 414 & $255 \mathrm{a}$ & $240 c$ & $244 \mathrm{~b}$ & $237 d$ & $236 d$ & $224 \mathrm{e}$ & $235 d$ \\
\hline MR11004 & 414 & $413 a$ & $391 \mathrm{c}$ & $397 \mathrm{~b}$ & $383 d$ & $384 \mathrm{~d}$ & $364 \mathrm{e}$ & $389 c$ \\
\hline SR11004 & 414 & $313 a$ & $298 \mathrm{~b}$ & $299 \mathrm{~b}$ & $298 \mathrm{~b}$ & 292 c & $284 \mathrm{~d}$ & 297 bc \\
\hline UR11004 & 414 & $703 \mathrm{~b}$ & $747 \mathrm{a}$ & $633 \mathrm{e}$ & $627 \mathrm{e}$ & $681 c$ & $658 d$ & $666 d$ \\
\hline $\begin{array}{l}\text { a TeeJet Techno } \\
\text { b Greenleaf Tec } \\
\text { c Wilger Indust } \\
\text { d Pentair Hypro } \\
\text { e Means within } \\
\text { configuration }\end{array}$ & $\begin{array}{l}\text { logies, Spraying Sys } \\
\text { hnologies, Covingto } \\
\text { ies Ltd., Lexington, } \\
\text { SHURflo plc., Minn } \\
\text { a gauge pressure an } \\
\text { with no solenoid va }\end{array}$ & $\begin{array}{l}\text { ns Co., G } \\
\text { A, USA. } \\
\text {, USA. } \\
\text { olis, MN, } \\
\text { ozzle wit } \\
\text { equippe }\end{array}$ & ame lett & ot signi & differer & .05). Sta & duty cy & ers to a sprayer \\
\hline
\end{tabular}

Driftable fines emitted from venturi nozzles were inversely proportional across duty cycles (Table 6). These droplet size patterns were expected according to the nozzle manufacturer's catalogues. For reference, the spray classifications were Coarse, Coarse, Very Coarse, Very Coarse, Very Coarse, Extremely Coarse, and Ultra Coarse for the AM11002, GAT11004, AMDF11004, AM11004, AMDF11008, AITTJ-6011004, and TTI11004 nozzles, respectively, at $276 \mathrm{kPa}$.

The addition of the solenoid valve to the spray system had variable effects on the droplet size distributions from venturi nozzles. The AITTJ-6011004, AMDF11008, and TTI11004 had greater droplet sizes and reduced or equal driftable fines across gauge pressures when the solenoid valve was operated at a $100 \%$ duty cycle compared to the standard configuration (no solenoid valve equipped). This is likely due to an additional restriction or elongated flow path within dual-fan and deflector-type venturi nozzles compared to other nozzles resulting in reduced pressure at the nozzle exit. Previous research corroborates this theory as reductions in droplet velocity from these nozzles were observed when a solenoid valve was equipped and operated at a $100 \%$ duty cycle (Butts et al., 2017).

The $D_{\mathrm{v} 0.1}, D_{\mathrm{v} 0.9}$, and driftable fines from venturi nozzles followed similar trends as model predictions of the $D_{\mathrm{v} 0.5}$ previously discussed. Typically, as duty cycle decreased, the $\mathrm{D}_{\mathrm{v} 0.1}$ and $\mathrm{D}_{\mathrm{v} 0.9}$ increased, and the driftable fines decreased across venturi nozzles and within gauge pressures. The average increase in $D_{\mathrm{v} 0.1}$ and $D_{\mathrm{v} 0.9}$ was $5.6 \%$ and $6.7 \%$, respectively, 
Table 5 - Droplet size data such that $90 \%$ of the spray volume is contained in droplets of lesser diameter $\left(D_{\mathrm{v} 0.9}\right)$ for water impacted by duty cycle for nozzle and pressure combinations.

Nozzle

$\mathrm{D}_{\mathrm{v} 0.9}$

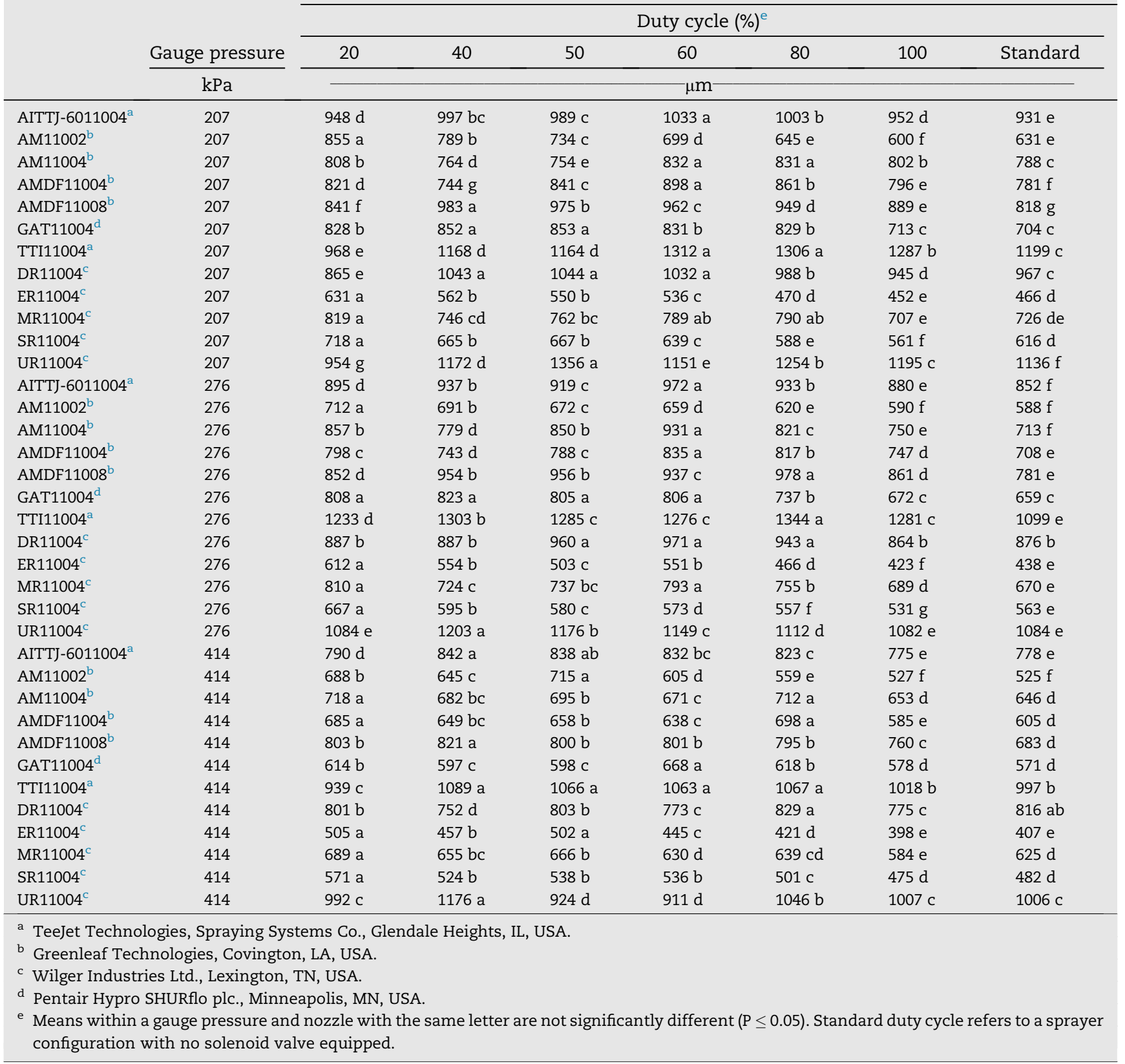

across venturi nozzles and within gauge pressures when duty cycle was decreased from 100 to $40 \%$. The effect of pulsing caused complex fluctuations in the droplet diameters across gauge pressures and venturi nozzles as the $\mathrm{D}_{\mathrm{v} 0.9}$ ranged from a decrease of $10.2 \%$ to an increase of $24.0 \%$ when duty cycle was reduced from 100 to $40 \%$. The general trend would indicate particle drift potential would decrease slightly from a pulsing PWM sprayer operated with venturi nozzles; however, due to the extreme fluctuations of the droplet size distributions and driftable fines emitted from venturi nozzles across a range of duty cycles and gauge pressures, this conclusion cannot be drawn with any certainty. Greater variability within venturi nozzle droplet size distribution measurements compared to non-venturi nozzles was also noted in previous research (Etheridge et al., 1999; Miller \& Butler Ellis, 2000). The variability resulted in negative effects on spray pattern (Ayers, Rogowski, \& Kimble, 1990) and decreased weed control (Etheridge, Hart, Hayes, \& Mueller, 2001). The unpredictable nature of droplet size distributions when affected by pulsing venturi nozzles is simply unacceptable for the optimization and homogenization of PWM sprays.

\subsubsection{Non-venturi nozzles}

Polynomial regressions established for non-venturi nozzles (DR11004, ER11004, MR11004, SR11004, and UR11004) to predict the effect of duty cycle on the $D_{\mathrm{v} 0.5}$ for each gauge 
Table 6 - Percent of spray volume less than $150 \mu \mathrm{m}$ (driftable fines) for water as impacted by duty cycle for each nozzle and pressure combination.

\begin{tabular}{|c|c|c|c|c|c|c|c|c|}
\hline \multirow[t]{3}{*}{ Nozzle } & & \multicolumn{7}{|c|}{ Driftable fines } \\
\hline & \multirow[b]{2}{*}{ Gauge pressure } & \multicolumn{7}{|c|}{ Duty cycle $(\%)^{e}$} \\
\hline & & 20 & 40 & 50 & 60 & 80 & 100 & Standard \\
\hline & $\mathrm{kPa}$ & \multicolumn{7}{|c|}{$\%-$} \\
\hline AITTJ-6011004 ${ }^{\mathrm{a}}$ & 207 & $0.09 \mathrm{c}$ & $0.54 \mathrm{~b}$ & $0.56 \mathrm{~b}$ & $0.55 \mathrm{~b}$ & $0.63 \mathrm{~b}$ & $0.71 \mathrm{ab}$ & $0.87 a$ \\
\hline AM11002 & 207 & $2.90 \mathrm{~d}$ & $2.62 \mathrm{f}$ & $2.78 \mathrm{e}$ & $3.33 \mathrm{c}$ & $3.97 \mathrm{~b}$ & $4.46 \mathrm{a}$ & $3.23 \mathrm{c}$ \\
\hline AM11004 & 207 & $2.27 \mathrm{~b}$ & $2.55 \mathrm{a}$ & $2.60 \mathrm{a}$ & $2.16 \mathrm{c}$ & $1.97 \mathrm{~d}$ & $2.33 \mathrm{~b}$ & $1.79 \mathrm{e}$ \\
\hline AMDF11004 & 207 & $2.12 \mathrm{~b}$ & $2.32 \mathrm{ab}$ & $2.17 \mathrm{~b}$ & $2.11 \mathrm{~b}$ & $2.15 \mathrm{~b}$ & $2.52 \mathrm{a}$ & $2.13 \mathrm{~b}$ \\
\hline AMDF11008 & 207 & $1.34 \mathrm{c}$ & $1.18 \mathrm{e}$ & $1.21 \mathrm{e}$ & $1.27 \mathrm{~d}$ & $1.31 \mathrm{~cd}$ & $1.43 \mathrm{~b}$ & $1.48 \mathrm{a}$ \\
\hline GAT11004 & 207 & $1.66 \mathrm{c}$ & $1.80 \mathrm{c}$ & $1.74 \mathrm{c}$ & $1.79 \mathrm{c}$ & $1.94 \mathrm{bc}$ & $2.91 \mathrm{a}$ & $2.16 \mathrm{~b}$ \\
\hline TTI11004 & 207 & $0.15 b$ & $0.33 \mathrm{ab}$ & $0.34 \mathrm{a}$ & $0.23 a b$ & $0.24 \mathrm{ab}$ & $0.25 \mathrm{ab}$ & $0.27 \mathrm{ab}$ \\
\hline DR11004 & 207 & $1.45 \mathrm{a}$ & $1.11 \mathrm{c}$ & $1.07 \mathrm{c}$ & $1.06 \mathrm{c}$ & $1.13 \mathrm{c}$ & $1.31 \mathrm{~b}$ & $0.77 \mathrm{~d}$ \\
\hline ER11004 & 207 & $11.78 \mathrm{e}$ & $14.03 \mathrm{~cd}$ & $14.36 \mathrm{c}$ & $14.45 \mathrm{bc}$ & $15.17 \mathrm{~b}$ & $16.60 \mathrm{a}$ & $13.56 \mathrm{~d}$ \\
\hline MR11004 & 207 & $3.16 \mathrm{bc}$ & $3.44 \mathrm{~b}$ & $2.98 \mathrm{c}$ & $3.12 \mathrm{bc}$ & $3.27 \mathrm{bc}$ & $4.11 \mathrm{a}$ & $2.24 \mathrm{~d}$ \\
\hline SR11004 & 207 & $6.18 \mathrm{e}$ & $7.14 \mathrm{~d}$ & $7.01 \mathrm{~d}$ & $7.55 \mathrm{c}$ & $7.92 \mathrm{~b}$ & $8.90 \mathrm{a}$ & $5.60 \mathrm{f}$ \\
\hline UR11004 & 207 & $0.73 \mathrm{a}$ & $0.52 \mathrm{~b}$ & $0.37 d$ & $0.50 \mathrm{~b}$ & $0.39 \mathrm{~d}$ & $0.45 c$ & $0.30 \mathrm{e}$ \\
\hline AITTJ-6011004 & 276 & $0.74 \mathrm{f}$ & $0.86 \mathrm{e}$ & $0.92 \mathrm{de}$ & $0.97 \mathrm{~d}$ & $1.12 \mathrm{c}$ & $1.21 \mathrm{~b}$ & $1.36 \mathrm{a}$ \\
\hline AM11002 & 276 & $4.49 \mathrm{~d}$ & $4.51 \mathrm{~d}$ & $4.72 \mathrm{c}$ & $4.77 \mathrm{c}$ & $5.03 \mathrm{~b}$ & $5.52 \mathrm{a}$ & $5.08 \mathrm{~b}$ \\
\hline AM11004 & 276 & $2.09 \mathrm{e}$ & $2.61 \mathrm{~d}$ & $2.70 \mathrm{~cd}$ & $2.61 \mathrm{~d}$ & $2.78 \mathrm{bc}$ & $2.88 \mathrm{ab}$ & $2.92 \mathrm{a}$ \\
\hline AMDF11004 & 276 & $2.90 \mathrm{e}$ & $3.12 \mathrm{~d}$ & $3.32 \mathrm{bc}$ & $3.18 \mathrm{~cd}$ & $3.20 \mathrm{~cd}$ & $3.72 \mathrm{a}$ & $3.39 \mathrm{~b}$ \\
\hline AMDF11008 & 276 & $1.45 \mathrm{~d}$ & $1.73 \mathrm{c}$ & $1.53 \mathrm{~d}$ & $1.63 c$ & $1.70 \mathrm{c}$ & $1.97 \mathrm{~b}$ & $2.36 \mathrm{a}$ \\
\hline GAT11004 $^{\mathrm{d}}$ & 276 & $1.94 \mathrm{f}$ & $2.08 \mathrm{e}$ & $2.15 \mathrm{de}$ & $2.21 \mathrm{~d}$ & $2.60 \mathrm{c}$ & $3.79 \mathrm{a}$ & $3.49 \mathrm{~b}$ \\
\hline TTI11004 ${ }^{a}$ & 276 & $0.01 \mathrm{~d}$ & $0.25 c$ & $0.25 c$ & $0.25 c$ & $0.25 c$ & $0.29 b$ & $0.48 \mathrm{a}$ \\
\hline DR11004 & 276 & $1.32 \mathrm{~d}$ & $1.51 \mathrm{c}$ & $1.52 \mathrm{c}$ & $1.61 \mathrm{~b}$ & $1.65 \mathrm{~b}$ & $1.81 \mathrm{a}$ & $1.28 \mathrm{~d}$ \\
\hline ER11004 & 276 & $13.67 \mathrm{~d}$ & $16.09 \mathrm{c}$ & $17.32 \mathrm{~b}$ & $14.26 \mathrm{~d}$ & $17.28 \mathrm{~b}$ & $19.32 \mathrm{a}$ & $16.90 \mathrm{bc}$ \\
\hline MR11004 & 276 & $2.90 \mathrm{e}$ & $3.49 \mathrm{~d}$ & $3.47 \mathrm{~d}$ & $3.78 \mathrm{c}$ & $4.14 \mathrm{~b}$ & $4.65 \mathrm{a}$ & $3.90 \mathrm{c}$ \\
\hline SR11004 & 276 & $8.11 \mathrm{e}$ & $9.09 \mathrm{~d}$ & $9.63 c$ & $9.58 \mathrm{c}$ & $10.21 \mathrm{~b}$ & $11.05 \mathrm{a}$ & $8.15 \mathrm{e}$ \\
\hline UR11004 & 276 & $0.01 \mathrm{f}$ & $0.49 \mathrm{~d}$ & $0.52 c$ & $0.55 \mathrm{~b}$ & $0.57 \mathrm{~b}$ & $0.64 \mathrm{a}$ & $0.44 \mathrm{e}$ \\
\hline AITTJ-6011004 & 414 & $1.66 \mathrm{f}$ & $1.90 \mathrm{e}$ & $1.91 \mathrm{e}$ & $2.04 \mathrm{~d}$ & $2.34 \mathrm{c}$ & $2.71 \mathrm{~b}$ & $3.03 \mathrm{a}$ \\
\hline AM11002 & 414 & $7.62 \mathrm{e}$ & $8.48 \mathrm{c}$ & $7.89 \mathrm{~d}$ & $8.52 c$ & $9.14 b$ & $9.93 \mathrm{a}$ & $8.50 \mathrm{c}$ \\
\hline AM11004 & 414 & $5.07 \mathrm{c}$ & $5.86 \mathrm{ab}$ & $6.14 \mathrm{a}$ & $5.90 \mathrm{ab}$ & $5.72 \mathrm{~b}$ & $6.18 \mathrm{a}$ & $5.22 \mathrm{c}$ \\
\hline AMDF11004 ${ }^{b}$ & 414 & $5.35 \mathrm{~d}$ & $5.89 c$ & $6.10 \mathrm{bc}$ & $6.40 \mathrm{~b}$ & $6.33 \mathrm{bc}$ & $7.03 \mathrm{a}$ & $6.43 \mathrm{~b}$ \\
\hline AMDF11008 ${ }^{b}$ & 414 & $3.18 \mathrm{f}$ & $3.75 \mathrm{e}$ & $3.92 \mathrm{de}$ & $3.95 \mathrm{~cd}$ & $4.13 \mathrm{c}$ & $4.38 \mathrm{~b}$ & $4.75 \mathrm{a}$ \\
\hline GAT11004 & 414 & $6.45 \mathrm{a}$ & $5.31 \mathrm{c}$ & $4.97 \mathrm{~d}$ & $4.96 \mathrm{~d}$ & $5.54 \mathrm{bc}$ & $5.78 \mathrm{~b}$ & $6.55 \mathrm{a}$ \\
\hline TTI11004 & 414 & $0.81 \mathrm{c}$ & $0.92 \mathrm{bc}$ & $0.95 a b c$ & $0.95 a b c$ & $0.94 \mathrm{abc}$ & $1.04 \mathrm{ab}$ & $1.08 \mathrm{a}$ \\
\hline DR11004 & 414 & $2.64 \mathrm{~d}$ & $2.94 \mathrm{c}$ & $3.08 \mathrm{~b}$ & $2.98 \mathrm{bc}$ & $3.09 \mathrm{~b}$ & $3.41 \mathrm{a}$ & $1.95 \mathrm{e}$ \\
\hline ER11004 & 414 & $21.72 \mathrm{~d}$ & $23.82 \mathrm{~b}$ & $22.93 \mathrm{c}$ & $23.86 \mathrm{~b}$ & $24.25 \mathrm{~b}$ & $25.58 \mathrm{a}$ & $22.22 \mathrm{~d}$ \\
\hline MR11004 & 414 & $5.75 \mathrm{~d}$ & $6.52 c$ & $6.71 c$ & $6.79 \mathrm{bc}$ & 7.02 b & $7.76 \mathrm{a}$ & $5.34 \mathrm{e}$ \\
\hline SR11004 & 414 & $13.35 \mathrm{bc}$ & $14.15 \mathrm{~b}$ & $14.26 \mathrm{ab}$ & $13.93 \mathrm{~b}$ & $14.58 \mathrm{ab}$ & $15.42 \mathrm{a}$ & $12.17 \mathrm{c}$ \\
\hline UR11004 & 414 & $1.06 \mathrm{ab}$ & $0.86 \mathrm{bc}$ & $1.05 \mathrm{ab}$ & $1.05 \mathrm{ab}$ & $1.04 \mathrm{ab}$ & $1.14 \mathrm{a}$ & $0.70 \mathrm{c}$ \\
\hline \multicolumn{9}{|c|}{ a TeeJet Technologies, Spraying Systems Co., Glendale Heights, IL, USA. } \\
\hline \multicolumn{9}{|c|}{ b Greenleaf Technologies, Covington, LA, USA. } \\
\hline \multicolumn{9}{|c|}{ c Wilger Industries Ltd., Lexington, TN, USA. } \\
\hline \multicolumn{9}{|c|}{ d Pentair Hypro SHURflo plc., Minneapolis, MN, USA. } \\
\hline $\begin{array}{l}\text { e Means within } \\
\text { configuration }\end{array}$ & th no solenoid val & equippe & - & triani & & & & \\
\hline
\end{tabular}

pressure are presented in Fig. 4. The resulting model parameters and $r^{2}$ values are presented in Table 2. Similar to venturi nozzles, as duty cycle decreased, droplet size increased across non-venturi nozzles (Fig. 4). The non-venturi nozzles required polynomial regressions, similar to the venturi nozzles, which may be an indication that more complex models are needed to appropriately fit droplet size data as affected by pulsing with current nozzle technologies, such as pre-orifice and venturi type nozzles, in contrast to conclusions from previous research using only non-venturi nozzles with no pre-orifice (Giles \& Comino, 1990). On average, non-venturi models predicted an increase in $D_{\mathrm{v} 0.5}$ as duty cycle decreased from 100 to $40 \%$ with estimated increases in $D_{\mathrm{v} 0.5}$ of $0.68,0.62$, and $0.34 \mu \mathrm{m}$ for every $1 \%$ decrease in duty cycle for 207,276 , and $414 \mathrm{kPa}$ gauge pressures, respectively. These increases in droplet size were smaller than those caused by pulsing venturi nozzles; therefore, non-venturi nozzles stabilized the droplet size distributions more than venturi nozzles across a range of duty cycles and would be the preferred nozzle on PWM sprayers. Similar to venturi nozzles, although $r^{2}$ values decreased as gauge pressure increased, the increase in gauge pressure buffered the pulsing effect on droplet size, further 
Table 7 - Average nozzle tip pressure over five seconds for water as impacted by nozzle for each gauge pressure and duty cycle combination.

Nozzle

Average nozzle tip pressure

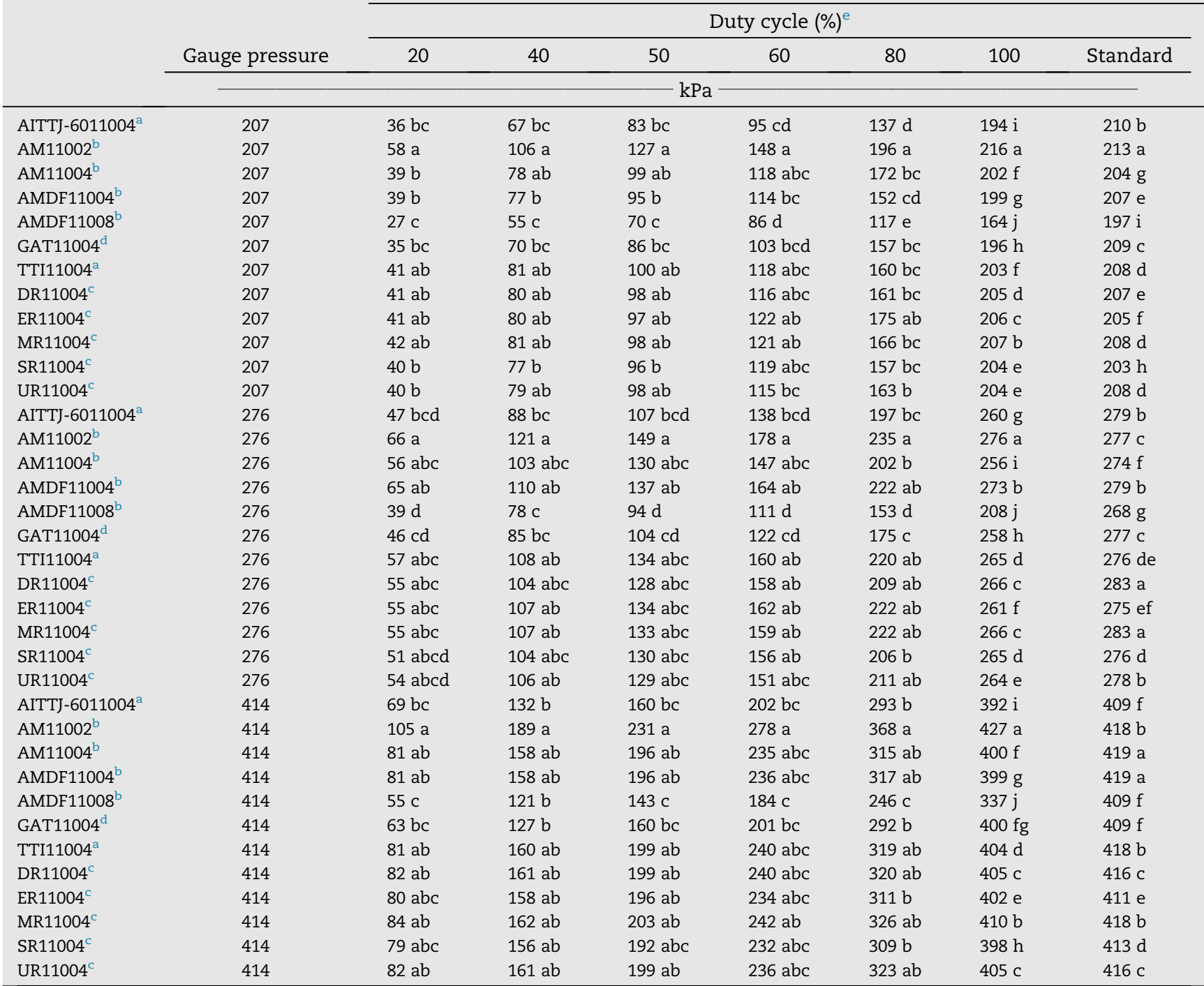

a TeeJet Technologies, Spraying Systems Co., Glendale Heights, IL, USA.

b Greenleaf Technologies, Covington, LA, USA.

c Wilger Industries Ltd., Lexington, TN, USA.

d Pentair Hypro SHURflo plc., Minneapolis, MN, USA.

e Means within a gauge pressure and duty cycle with the same letter are not significantly different $(\mathrm{P} \leq 0.05)$. Standard duty cycle refers to a sprayer configuration with no solenoid valve equipped.

validating PWM sprayers should be operated at greater gauge pressures $(\geq 276 \mathrm{kPa}$ ) as much as drift mitigation efforts allow.

The $\mathrm{D}_{\mathrm{v} 0.1}, \mathrm{D}_{\mathrm{v} 0.5}, \mathrm{D}_{0.9}$, and driftable fines emitted from nonventuri nozzles as affected by PWM duty cycle are presented in Table 3 through 6 . Across duty cycles, the droplet size distributions from non-venturi nozzles followed the pattern (from smallest to greatest): ER11004 < SR11004 < MR11004 < DR11004 < UR11004 (Tables 3-5). Driftable fines emitted from non-venturi nozzles followed the inverse pattern across duty cycles (Table 6). These trends were expected according to the nozzle manufacturer's catalogue. For reference, the spray classifications were Medium, Medium, Coarse, Extremely Coarse, and Extremely Coarse for the
ER11004, SR11004, MR11004, DR11004, and UR11004 nozzles, respectively, at $276 \mathrm{kPa}$. In previous PWM literature, only nonventuri nozzles with no pre-orifice were evaluated (Giles \& Comino, 1990; Giles et al., 1996, pp. 23-26). For the nonventuri nozzles evaluated in this research, four out of five (SR11004, MR11004, DR11004, and UR11004) had pre-orifices, and little to no difference was observed in the droplet size trends when pulsed between the non-venturi nozzles with pre-orifices and the non-venturi nozzle without a pre-orifice (ER11004).

The addition of an inline solenoid valve caused a decrease in droplet size when operated at a $100 \%$ duty cycle compared to the standard configuration (no solenoid valve equipped) within 
gauge pressures and across most non-venturi nozzles. This result was peculiar as the nozzle tip pressure data, discussed in detail later in this manuscript, revealed a decrease in pressure across the solenoid valve. Flow rates of non-venturi nozzles across gauge pressures were measured to determine if flow rates were increasing through a solenoid valve to explain the droplet size decrease (data not shown). The addition of a solenoid valve operated at a $100 \%$ duty cycle decreased flow rate by approximately $5 \%$ compared to the standard configuration, matching the nozzle tip pressure reductions observed from the addition of a solenoid valve (Table 7). Therefore, this does not explain the decrease in droplet size from non-venturi nozzles
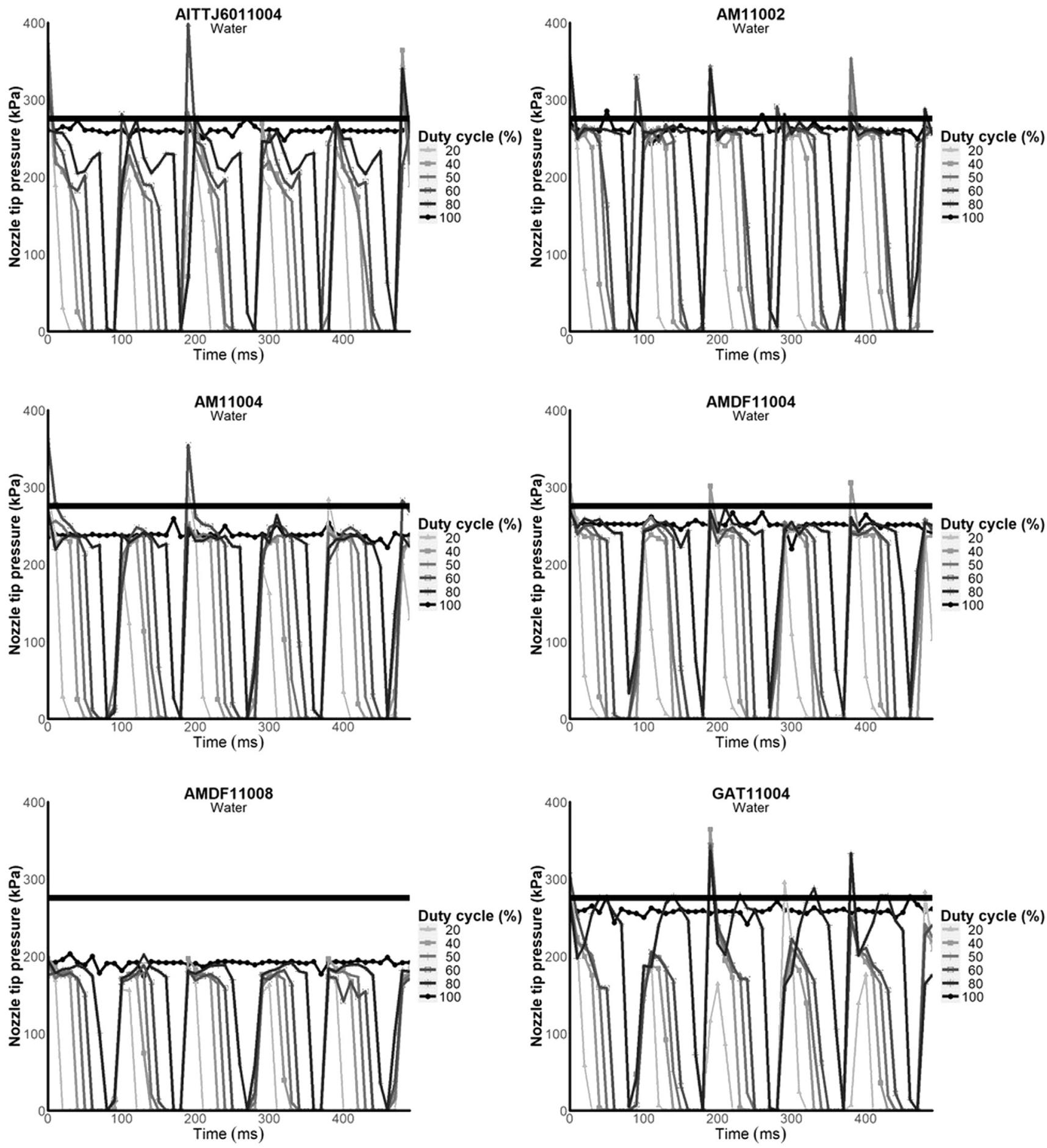

Fig. 5 - Fluctuations in nozzle tip pressure $(\mathrm{kPa})$ over $0.5 \mathrm{~s}$ for a gauge pressure of $276 \mathrm{kPa}$ with water spray solution as influenced by duty cycle for the AITTJ-6011004 (top left), AM11002 (top right), AM11004 (middle left), AMDF11004 (middle right), AMDF11008 (bottom left), and GAT11004 (bottom right) nozzles. The solid black bar indicates the $276 \mathrm{kPa}$ gauge pressure. 
operated at a $100 \%$ duty cycle compared to a standard configuration and further research should be conducted to identify the underlying cause. Overall, the decrease in droplet size indicates PWM sprayers operating with non-venturi nozzles at high duty cycles increase spray drift potential slightly compared to conventional sprayers. However, this increase in spray drift potential is minimal, especially when compared to the drift potential increases observed from conventional sprayers implementing similar flow rate changes (Giles, Downey, Kolb, \& Grimm, 2003).

The $D_{\mathrm{v} 0.1}$ and $\mathrm{D}_{0.9}$ generally increased as duty cycle decreased across non-venturi nozzles and gauge pressures similar to the model predictions for the $D_{\mathrm{v} 0.5}$. The $D_{\mathrm{v} 0.1}$ and $\mathrm{D}_{\mathrm{v} 0.9}$ increased by an average of 6.0 and $9.6 \%$, respectively, within gauge pressures and across non-venturi nozzles when the duty cycle was reduced from 100 to $40 \%$. The non-venturi
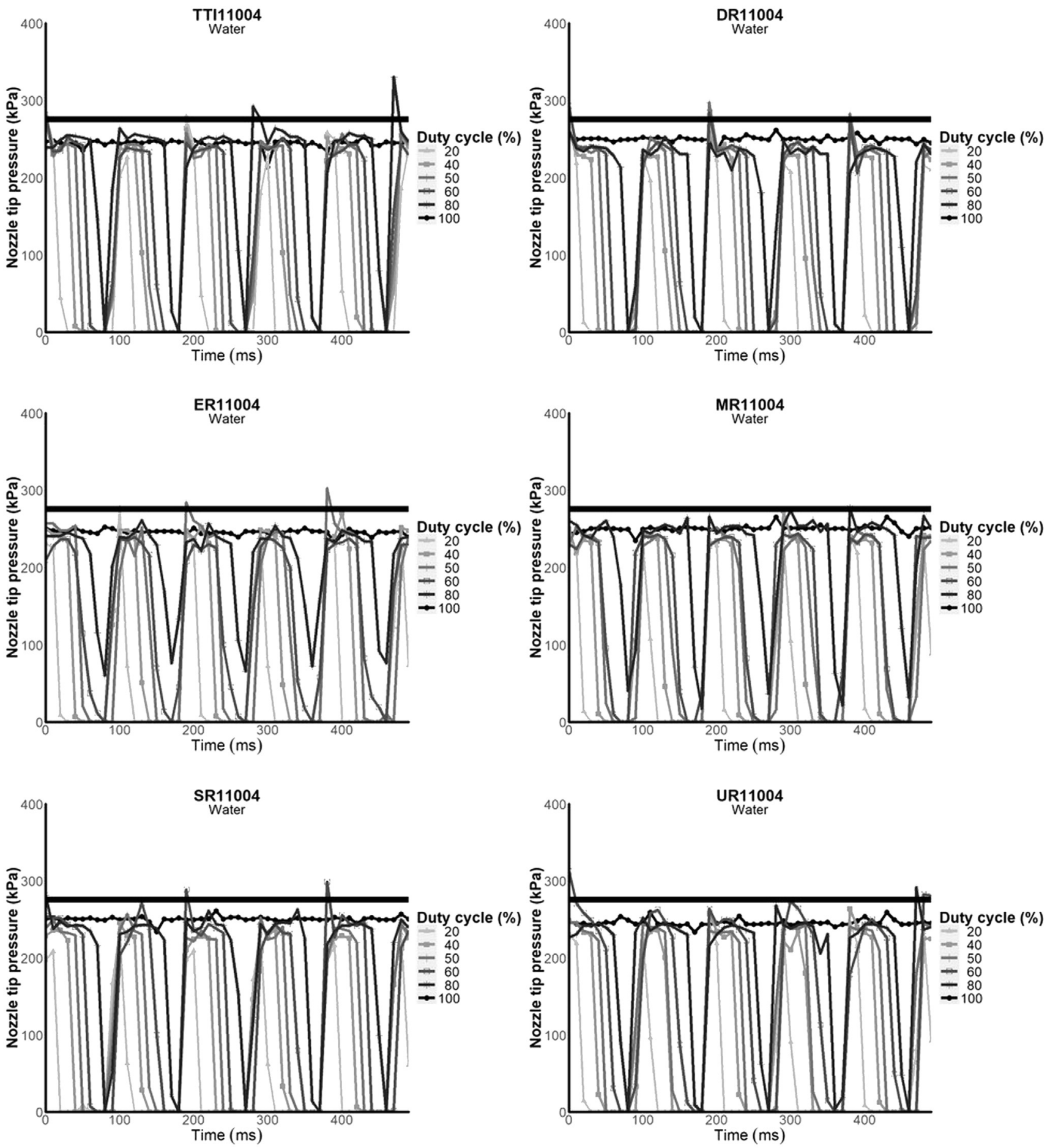

Fig. 6 - Fluctuations in nozzle tip pressure $(\mathrm{kPa})$ over $0.5 \mathrm{~s}$ for a gauge pressure of $276 \mathrm{kPa}$ with water spray solution as influenced by duty cycle for the TTI11004 (top left), DR11004 (top right), ER11004 (middle left), MR11004 (middle right), SR11004 (bottom left), and UR11004 (bottom right) nozzles. The solid black bar indicates the $276 \mathrm{kPa}$ gauge pressure. 
nozzle droplet size distributions fluctuated when pulsed, but not as great as the venturi nozzles, as the $D_{\mathrm{v} 0.9}$ values ranged from a decrease of $3.1 \%$ to an increase of $23.6 \%$ when the duty cycle was reduced from 100 to $40 \%$. The driftable fines were reduced by $0.0-3.2$ percentage points across non-venturi nozzles and within gauge pressures as the duty cycle decreased from 100 to $40 \%$ indicating the pulsing of PWM sprayers can reduce particle drift potential. Overall, droplet size distributions from non-venturi nozzles were more stable and homogenous when pulsed compared to venturi nozzles,
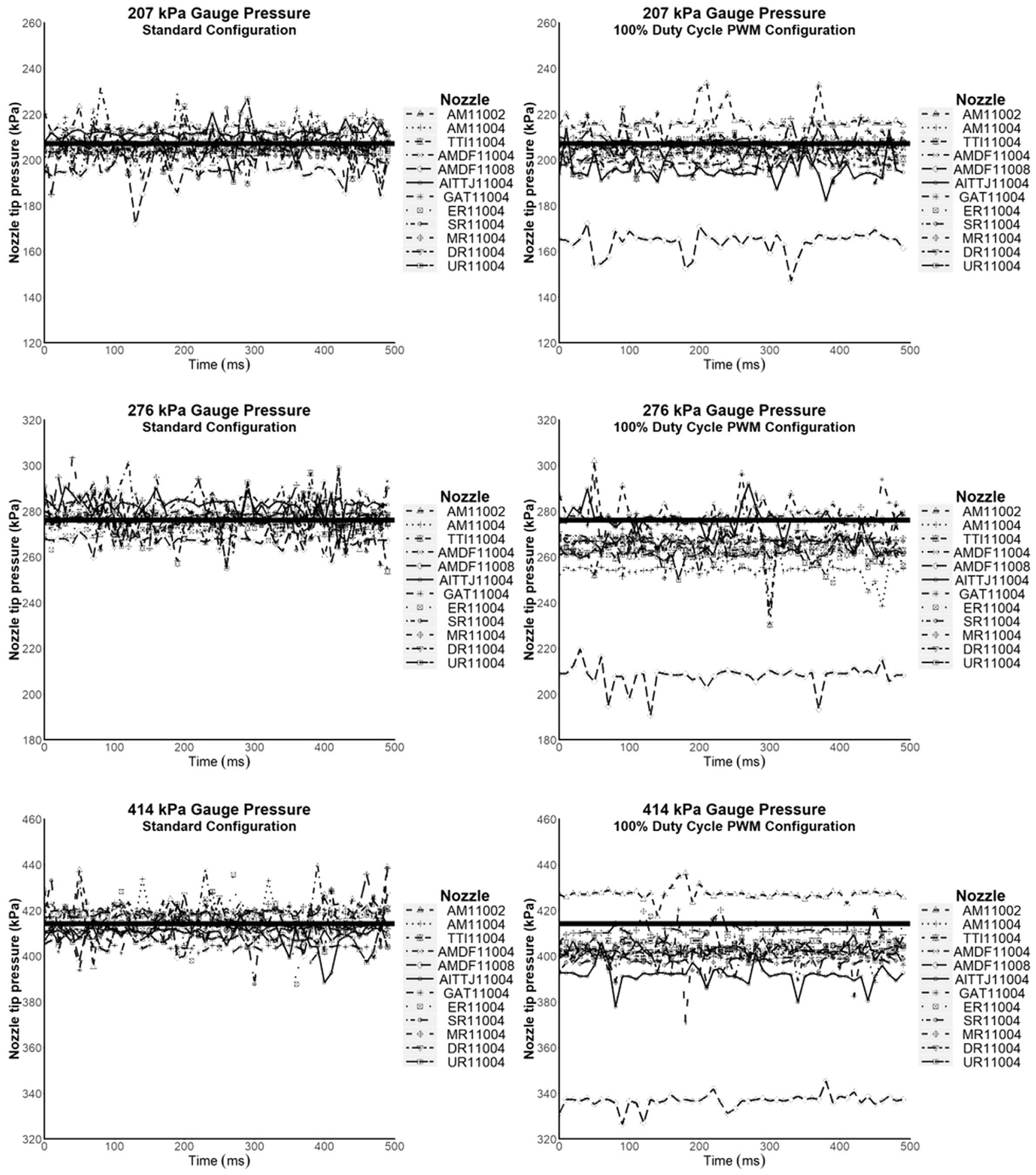

Fig. 7 - Nozzle tip pressure of 12 nozzles when spraying water in a standard nozzle body configuration (no solenoid valve) at $207 \mathrm{kPa}$ (top left), $276 \mathrm{kPa}$ (middle left), and $414 \mathrm{kPa}$ (bottom left) and at a 100\% duty cycle in a pulsing nozzle body configuration (with solenoid valve) at $207 \mathrm{kPa}$ (top right), $276 \mathrm{kPa}$ (middle right), and $414 \mathrm{kPa}$ (bottom right). The solid black bar indicates the respective gauge pressure. 
and the addition of a pre-orifice had little to no impact on the droplet size trends observed across PWM duty cycles. Therefore, non-venturi nozzles with or without pre-orifices are recommended for use on PWM sprayers to stabilize droplet size distributions across a range of duty cycles, and a $40 \%$ duty cycle or greater should be utilized to optimize and homogenize PWM pesticide applications, especially for site-specific pest management strategies requiring an explicit droplet size.

\subsection{Nozzle tip pressure}

Visual assessments of nozzle tip pressure patterns across duty cycles revealed minimal deviations from the square wave PWM electrical signal pattern due to gauge pressure changes. Nozzle tip pressure measurements over time at the $276 \mathrm{kPa}$ gauge pressure are presented in Figs. 5 and 6. They illustrate PWM duty cycles operating at the $10 \mathrm{~Hz}$ frequency and that nozzle tip pressures do not follow the square wave electrical signal pattern explicitly, especially across nozzle types (Figs. 5 and 6). Some of the pressure measurement variability can be attributed to the single nozzle/spray solution supply line used for testing (Fig. 1). Commercial systems buffer this effect by placing multiple solenoid valves, operating on alternate frequencies, on a similar supply line or boom section (Mangus et al., 2017). Nozzle tip pressure peaks and valleys emerged for venturi nozzles, excluding the AMDF11008 and TTI11004, compared to non-venturi nozzles. Additionally, the AITTJ6011004 and GAT11004 venturi nozzles had severe deformities in nozzle tip pressure measurement patterns when pulsed. This is likely due to the nozzle design of each as the AITTJ-6011004 and GAT11004 have a single pre-orifice with dual fan exit orifices which is unique compared to other nozzles tested. Although these pressure fluctuation deformities did not influence droplet size to a great extent, spray pattern could be highly affected, and should be evaluated in future research.

The average nozzle tip pressure measurement trends across duty cycle were unaffected by gauge pressure (Table 7). Nozzle design and orifice size impacted the nozzle tip pressure measurements across gauge pressures and duty cycles. When the PWM duty cycle was reduced from $100 \%$ to a specific duty cycle, the average nozzle tip pressure reduction should have been equivalent to the duty cycle reduction (i.e. if the duty cycle were reduced from 100 to $50 \%$, the average nozzle tip pressure at the $50 \%$ duty cycle should be half of the nozzle tip pressure at the $100 \%$ duty cycle). When nozzle orifice size decreased (AM11002), the percent change in average nozzle tip pressure was less than expected (54\%) across gauge pressures if duty cycle was reduced by $60 \%$. In contrast, when nozzle orifice size increased (AMDF11008), the percent change in average nozzle tip pressure was greater than expected (64\%) across gauge pressures if duty cycle was reduced by $60 \%$. The AITTJ-6011004 and GAT11004 nozzles again had larger disturbances in their nozzle tip pressure patterns compared to other nozzles. The percent change in average nozzle tip pressure for the AITTJ-6011004 and GAT11004 was greater than expected, $66 \%$ for both nozzles across gauge pressures, if duty cycle was reduced by $60 \%$. Other nozzles tested had a percent change in average nozzle tip pressure of $60 \%$ across gauge pressures if duty cycle was reduced $60 \%$.
Measurements further revealed a reduction in nozzle tip pressure as orifice size increased and when the dual fan, single pre-orifice venturi nozzles (AITTJ-6011004 and GAT11004) were equipped and operated at a $100 \%$ duty cycle compared to a standard configuration with no solenoid valve equipped (Fig. 7). The AITTJ-6011004, AMDF11008, and GAT11004 had the lowest average nozzle tip pressures and the AM11002 had the greatest average nozzle tip pressure compared to other nozzles across gauge pressures when a solenoid valve was equipped. The greatest pressure reduction observed was for the AMDF11008 which had a loss in pressure of nearly $75 \mathrm{kPa}$. These pressure losses are likely produced by a restriction within the solenoid valve; therefore, maximum flow is restricted especially with greater orifice sizes (flow rates), and a low pressure area is created on the exit side of the solenoid. Commercial PWM systems adjust for this pressure loss with an increase in calculated duty cycle to maintain the appropriate output. However, applicators should make note of this pressure loss, as several negative impacts may arise from this finding: (1) the reduced pressure at the nozzle increases droplet size compared to what would be expected from the input gauge pressure, and reductions in biological efficacy may occur, especially in droplet size oriented site-specific pest management strategies; (2) if PWM sprayers were operated at low gauge pressures, the pressure loss may result in nozzles being operated below nozzle manufacturer's recommended pressure ranges; and (3) the reduced nozzle pressure may lead to incomplete pattern formation, especially when pulsed, resulting in reduced efficacy and inefficient applications.

\section{Conclusions}

The effectiveness of site-specific pest management strategies relies on two factors, (1) maximizing the biological effect, and (2) minimizing environmental contamination through offtarget spray movement. Spray droplet size is a critical component to influence these two factors simultaneously. If spray droplet size is to be optimized and homogenized across a PWM application, the following best use practices should be followed:

1. PWM sprayers should be operated at or above a $40 \%$ duty cycle. Droplet size was severely affected and the pattern of change was inconsistent when pulsed at the $20 \%$ duty cycle tested in this research.

2. PWM sprayers should be operated at or above $276 \mathrm{kPa}$ gauge pressure. This practice buffers the pulsing impact on droplet size and remains above nozzle manufacturers' recommended pressures due to the pressure loss across the solenoid valve.

3. Only non-venturi nozzles should be equipped and operated on PWM sprayers. These nozzle types, with and without pre-orifices, minimize variation in droplet size and nozzle tip pressure across duty cycles compared with venturi nozzles.

Applicators using a PWM sprayer should also acknowledge the pressure loss across the solenoid valve. The decreased pressure, especially for greater orifice size nozzles, could 
affect spray pattern and create coarser droplet sizes than desired for biological control. Further, as PWM duty cycle decreases, spray droplet size increases, thereby potentially impacting spray coverage and the resulting biological efficacy. Across non-venturi nozzles and gauge pressures, droplet size $\left(D_{\mathrm{v} 0.5}\right)$ increased by approximately $0.55 \mu \mathrm{m}$ for every $1 \%$ decrease in duty cycle. Spray solution changed the overall droplet sizes observed; however, the effect of pulsing had little to no impact on the droplet size trends observed across duty cycles for the solutions tested. Through these practices, applicators can increase the efficiency of PWM pesticide applications and reduce the risks of off-target spray particle movement by better understanding the complexities of spray applications.

\section{Acknowledgements}

The authors would like to thank Brian Finstrom and Capstan Ag Systems, Inc. for providing PWM equipment and technical support, Shane Forney for assistance with nozzle tip pressure measurements, and Jake Jungbluth for Arduino code assistance. The authors would also like to thank Greenleaf Technologies, Pentair Hypro, TeeJet Technologies, and Wilger Industries for supplying nozzles used in this research.

\section{R E F E R E N C E S}

Anglund, E. A., \& Ayers, P. D. (2003). Field evaluation of response times for a variable rate (pressure-based and injection) liquid chemical applicator. Applied Engineering for Agriculture, 19(3), 273-282. https://doi.org/10.13031/2013.13659.

ASABE. (2009). Spray nozzle classification by droplet spectra. St. Joseph, MI. https://doi.org/ANSI/ASAE S572.1.

Ayers, P. D., Rogowski, S. M., \& Kimble, B. L. (1990). An investigation of factors affecting sprayer control system performance. Applied Engineering for Agriculture, 6(6), 701-706. https://doi.org/10.13031/2013.26451.

Barnett, G. S., \& Matthews, G. A. (1992). Effect of different fan nozzles and spray liquids on droplet spectra with special reference to drift control. International Pest Control, 34(3), 81-85.

Bish, M. D., \& Bradley, K. W. (2017). Survey of Missouri pesticide applicator practices, knowledge, and perceptions. Weed Technology, 31, 165-177. https://doi.org/10.1017/wet.2016.27.

Bouse, L. F. (1994). Effect of nozzle type and operation on spray droplet size. Transactions of ASAE, 37(5), 1389-1400. https://doi. org/10.13031/2013.28219.

Bouse, L. F., Kirk, I. W., \& Bode, L. E. (1990). Effect of spray mixture on droplet size. Transaction of ASAE, 33(3), 783-788. https://doi. org/10.13031/2013.31401.

Bueno, M. R., da Cunha, J. P. A. R., \& de Santana, D. G. (2017). Assessment of spray drift from pesticide applications in soybean crops. Biosystems Engineering, 154, 35-45. https://doi. org/10.1016/j.biosystemseng.2016.10.017.

Butler Ellis, M. C., Swan, T., Miller, P. C. H., Waddelow, S., Bradley, A., \& Tuck, C. R. (2002). Design factors affecting spray characteristics and drift performance of air induction nozzles. Biosystems Engineering, 82(3), 289-296. https://doi.org/10.1006/ bioe.2002.0069.

Butler Ellis, M. C., Tuck, C. R., \& Miller, P. C. H. (1997). The effect of some adjuvants on sprays produced by agricultural flat fan nozzles. Crop Protection, 16(1), 41-50. https://doi.org/10.1016/ S0261-2194(96)00065-8.

Butts, T. R., Geyer, A. M., \& Kruger, G. R. (2015). Wide range of droplet size distributions from non-venturi nozzles (Vol. 70, p. 42). Indianapolis: Proceedings North Central Weed Science Society.

Butts, T. R., Hoffmann, W. C., Luck, J. D., \& Kruger, G. R. (2018). Droplet velocity from broadcast agricultural nozzles as influenced by pulse-width modulation. In B. K. Fritz, \& T. R. Butts (Eds.), Pesticide formulations and delivery systems: Innovative application, formulation, and adjuvant technologies Vol. 38 (pp. 24-52). West Conshohocken, PA: ASTM International. https://doi.org/10.1520/STP161020170192.

Capstan Ag Systems Inc. (2013). PinPoint Synchro product manual. Topeka. KS 66609 https://doi.org/PN-120156-001.

Chapple, A. C., Downer, R. A., \& Hall, F. R. (1993). Effects of spray adjuvants on swath patterns and droplet spectra for a flat-fan hydraulic nozzle. Crop Protection, 12(8), 579-590. https://doi. org/10.1016/0261-2194(93)90120-8.

Creech, C. F., Henry, R. S., Fritz, B. K., \& Kruger, G. R. (2015). Influence of herbicide active ingredient, nozzle type, orifice size, spray pressure, and Carrier volume rate on spray droplet size characteristics. Weed Technology, 29(2), 298-310. https:// doi.org/10.1614/WT-D-14-00049.1.

De Cock, N., Massinon, M., Salah, S. O. T., \& Lebeau, F. (2017). Investigation on optimal spray properties for ground based agricultural applications using deposition and retention models. Biosystems Engineering, 162, 99-111. https://doi.org/10. 1016/j.biosystemseng.2017.08.001.

Ebert, T. A., Taylor, R. A. J., Downer, R. A., \& Hall, F. R. (1999). Deposit structure and efficacy of pesticide application. 1: Interactions between deposit size, toxicant concentration and deposit number. Pesticide Science, 55, 783-792. https://doi.org/10.1002/ (SICI)1096-9063(199908)55:8<783::AID-PS973>3.0.CO;2-D.

Etheridge, R. E., Hart, W. E., Hayes, R. M., \& Mueller, T. C. (2001). Effect of venturi-type nozzles and application volume on postemergence herbicide efficacy. Weed Technology, 15(1), 75-80. https://doi.org/10.1614/0890-037X(2001)015[0075: EOVTNA]2.0.CO;2.

Etheridge, R. E., Womac, A. R., \& Mueller, T. C. (1999). Characterization of the spray droplet spectra and patterns of four venturi-type drift reduction nozzles. Weed Technology, 13(4), 765-770. https://doi.org/10.1017/S0890037X00042202.

Fritz, B. K., Hoffmann, W. C., Kruger, G. R., Henry, R. S., Hewitt, A. J., \& Czaczyk, Z. (2014). Comparison of drop size data from ground and aerial application nozzles at three testing laboratories. Atomization and Sprays, 24(2), 181-192. https://doi.org/10.1615/AtomizSpr.2013009668.

Giles, D. K. (2001). Pulsed sprays from oversized orifices for increased momentum and kinetic energy in depositing agricultural sprays. Atomization and Sprays, 11(6), 701-709. https://doi.org/10.1615/AtomizSpr.v11.i6.60.

Giles, D. K., \& Ben-Salem, E. (1992). Spray droplet velocity and energy in intermittent flow from hydraulic nozzles. Journal of Agricultural Engineering Research, 51, 101-112. https://doi.org/ 10.1016/0021-8634(92)80029-R.

Giles, D. K., \& Comino, J. A. (1989). Variable flow control for pressure atomization nozzles. Journal of Commerical Vehicles, SAE Transactions, 98(2), 237-249. https://doi.org/10.4271/ 891836.

Giles, D. K., \& Comino, J. A. (1990). Droplet size and spray pattern characteristics of an electronic flow controller for spray nozzles. Journal of Agricultural Engineering Research, 47, 249-267. https://doi.org/10.1016/0021-8634(90)80045-V.

Giles, D. K., Downey, D., Kolb, T., \& Grimm, J. G. (2003). Pulsewidth modulated sprays for flow rate and droplet size control: Spray dynamics and field performance. In R. Downer, J. Mueninghoff, \& G. Volgas (Eds.), Pesticide formulations and 
delivery systems: Meeting the challenges of the current crop protection industry Vol. 22, ASTM STP 1430. West Conshohocken, PA 19428-2959, USA: ASTM International, 100 Barr Harbor Drive, PO Box C700 https://doi.org/10.1520/ STP1430-EB.

Giles, D. K., Henderson, G. W., \& Funk, K. (1996). Digital control of flow rate and spray droplet size from agricultural nozzles for precision chemical application. Precision agriculture. Proceedings of the 3rd International Conference, Minneapolis, Minnesota, USA. Madison; USA: American Society of Agronomy. Retrieved from http://apps.webofknowledge.com/full_record.do?product=UA \&search $\_$mode $=$GeneralSearch $\& q i d=2 \&$ SID $=$ 4EDACoZqq5YtSSw3rL8\&page $=1 \&$ doc $=9$.

Grisso, R. D., Dickey, E. C., \& Schulze, L. D. (1989). The cost of misapplication of herbicides. Applied Engineering in Agriculture, 5(3), 344-347. https://doi.org/10.13031/2013.26525.

Henry, R. S., Kruger, G. R., Fritz, B. K., Hoffmann, W. C., \& Bagley, W. E. (2014). Measuring the effect of spray plume angle on the accuracy of droplet size data. In C. Sesa (Ed.), Pesticide formulations and delivery systems: Sustainability: Contributions from formulation technology (Vol. 33). West Conshohocken, PA: ASTM International. https://doi.org/10. 1520/STP156920120130.

Hewitt, A. J. (1997). Droplet size and agricultural spraying, Part I: Atomization, spray transport, deposition, drift, and droplet size measurement techniques. Atomization and Sprays, 7(3), 235-244. https://doi.org/10.1615/AtomizSpr.v7.i3.10.

Hoffmann, W. C., Fritz, B. K., \& Martin, D. E. (2011). Air and spray mixture temperature effects on atomization of agricultural sprays. Agricultural Engineering International: CIGR Journal, 13(1). Manuscript No. 1730.

Johnson, A. K., Roeth, F. W., Martin, A. R., \& Klein, R. N. (2006). Glyphosate spray drift management with drift-reducing nozzles and adjuvants. Weed Technology, 20(4), 893-897. https://doi.org/10.1614/WT-05-162.1.

Knoche, M. (1994). Effect of droplet size and carrier volume on performance of foliage-applied herbicides. Crop Protection, 13(3), 163-178. https://doi.org/10.1016/0261-2194(94)90075-2.

Luck, J. D., Pitla, S. K., Shearer, S. A., Mueller, T. G., Dillon, C. R., Fulton, J. P., et al. (2010a). Potential for pesticide and nutrient savings via map-based automatic boom section control of spray nozzles. Computers and Electronics in Agriculture, 70(1), 19-26. https://doi.org/10.1016/j.compag.2009.08.003.

Luck, J. D., Sharda, A., Pitla, S. K., Fulton, J. P., \& Shearer, S. A. (2011). A case study concerning the effects of controller response and turning movements on application rate uniformity with a self-propelled sprayer. Transactions of ASABE, 54(2), 423-431. https://doi.org/10.13031/2013.36445.

Luck, J. D., Zandonadi, R. S., Luck, B. D., \& Shearer, S. A. (2010b). Reducing pesticide over-application with map-based automatic boom section control on agricultural sprayers. Transactions of ASABE, 53(3), 685-690. https://doi.org/10.13031/2013.30060.

Mangus, D. L., Sharda, A., Engelhardt, A., Flippo, D., Strasser, R., Luck, J. D., et al. (2017). Analyzing the nozzle spray fan pattern of an agricultural sprayer using pulse-width modulation technology to generate an on-ground coverage map.
Transactions of ASABE, 60(2), 315-325. https://doi.org/10.13031/ trans.11835.

Matthews, G., Bateman, R., \& Miller, P. (2014). Pesticide application methods (4th ed.). Wiley-Blackwell.

Meyer, C. J., Norsworthy, J. K., Kruger, G. R., \& Barber, T. L. (2016). Effect of nozzle selection and spray volume on droplet size and efficacy of Engenia tank-mix combinations. Weed Technology, 30, 377-390. https://doi.org/10.1614/WT-D-1500141.1.

Miller, P. C. H., \& Butler Ellis, M. C. (2000). Effects of formulation on spray nozzle performance for applications from groundbased boom sprayers. Crop Protection, 19(8-10), 609-615. https://doi.org/10.1016/S0261-2194(00)00080-6.

Miller, P. C. H., \& Tuck, C. R. (2005). Factors influencing the performance of spray delivery systems: A review of recent developments. Journal of ASTM International, 2(6), 133-145. https://doi.org/10.1520/JAI12900.

Nuyttens, D., Baetens, K., De Schampheleire, M., \& Sonck, B. (2007). Effect of nozzle type, size and pressure on spray droplet characteristics. Biosystems Engineering, 97(3), 333-345. https:// doi.org/10.1016/j.biosystemseng.2007.03.001.

Nuyttens, D., De Schampheleire, M., Verboven, P., Brusselman, E., \& Dekeyser, D. (2009). Droplet size and velocity characteristics of agricultural sprays. Transactions of ASABE, 52(5), 1471-1480. https://doi.org/10.13031/2013.29127.

Ozkan, H. E. (1987). Sprayer performance evaluation with microcomputers. Applied Engineering in Agriculture, 3(1), 36-41. https://doi.org/10.13031/2013.26641.

Sharda, A., Fulton, J. P., McDonald, T. P., \& Brodbeck, C. J. (2011). Real-time nozzle flow uniformity when using automatic section control on agricultural sprayers. Computers and Electronics in Agriculture, 79(2), 169-179. https://doi.org/10.1016/ j.compag.2011.09.006.

Sharda, A., Luck, J. D., Fulton, J. P., McDonald, T. P., \& Shearer, S. A. (2013). Field application uniformity and accuracy of two rate control systems with automatic section capabilities on agricultural sprayers. Precision Agriculture, 14(3), 307-322. https://doi.org/10.1007/s11119-012-9296-z.

Stroup, W. W. (2013). Generalized linear mixed models: Modern concepts, methods and applications. CRC Press, Taylor \& Francis Group.

Womac, A. R., Melnichenko, G., Steckel, L. E., Montgomery, G., \& Hayes, R. M. (2016). Spray tip effect on glufosinate canopy deposits in Palmer amaranth (Amaranthus palmeri) for pulsewidth modulation versus air-induction technologies. Transaction of ASABE, 59(6), 1597-1608. https://doi.org/10. 13031/trans.59.11642.

Womac, A. R., Melnichenko, G., Steckel, L. E., Montgomery, G., Reeves, J., \& Hayes, R. M. (2017). Spray tip configurations with pulse-width modulation for glufosinate-ammonium deposits in Palmer amaranth (Amaranthus palmeri). Transactions of ASABE, 60(4), 1123-1136. https://doi.org/10.13031/trans.12137.

Young, B. W. (1990). Droplet dynamics in hydraulic spray clouds. In Tenth symposium on pesticide formulations and application systems (STP1078). West Conshohocken, PA: ASTM International. https://doi.org/10.1520/STP25378S. 